\title{
Constructing representations of Hecke algebras for complex reflection groups
}

\author{
Gunter Malle and Jean Michel
}

\begin{abstract}
We investigate the representations and the structure of Hecke algebras associated to certain finite complex reflection groups. We first describe computational methods for the construction of irreducible representations of these algebras, including a generalization of the concept of a $W$-graph to the situation of complex reflection groups. We then use these techniques to find models for all irreducible representations in the case of complex reflection groups of dimension at most three. Using these models we are able to verify some important conjectures on the structure of Hecke algebras.
\end{abstract}

\section{Introduction}

Let $W \leqslant \mathrm{GL}(V)$ be a finite irreducible group on a complex vector space $V$ generated by complex reflections; that is, $W$ is a finite complex reflection group. Let $R \subset W$ denote the set of reflections in $W$. For any reflection $s \in R$ let $H_{s} \subset V$ denote its hyperplane of fixed points on $V$. Then $V^{\text {reg }}:=V \backslash \bigcup_{s \in R} H_{s}$ is connected in the complex topology, and $W$ acts freely (and continuously) on $V^{\text {reg }}$ by the well-known theorem of Steinberg. The braid group associated to $(W, V)$ is the fundamental group

$$
B(W):=\pi_{1}\left(\bar{V}, \bar{x}_{0}\right)
$$

of the quotient $\bar{V}:=V^{\text {reg }} / W$ with respect to some base point $\bar{x}_{0} \in \bar{V}$.

Let $H$ be the reflecting hyperplane of some reflection of $W$. Then its stabilizer $W_{H}$ is cyclic, consisting solely of reflections (and the identity). The distinguished reflection $s_{H} \in W_{H}$ of $W_{H}$ is by definition the reflection whose non-trivial eigenvalue on $V$ equals $\exp (2 \pi i / d)$, where $d:=\left|W_{H}\right|$. Via the natural projection map from $B(W)$ onto $W$ induced by the quotient map $V^{\text {reg }} \rightarrow \bar{V}$, the distinguished reflection $s_{H}$ can be lifted to so-called braid reflections $\mathbf{s}$ in $B(W)$. For each reflection $s_{H}$ choose $d$ indeterminates $u_{s, 0}, \ldots, u_{s, d-1}$ such that $u_{s, j}=u_{t, j}$ if $s, t$ are conjugate in $W$. We write $\mathbf{u}$ for the collection of these indeterminates, and let $A:=\mathbb{Z}\left[\mathbf{u}, \mathbf{u}^{-1}\right]$. The generic cyclotomic Hecke algebra associated to $W$ with parameters $\mathbf{u}$ is the quotient

$$
\mathcal{H}(W, \mathbf{u}):=A B(W) / I
$$

of the group algebra $A B(W)$ of the braid group $B(W)$ by the ideal $I$ generated by the $\prod_{i=0}^{d-1}\left(\mathbf{s}-u_{s, i}\right)$, where $s$ runs over the distinguished reflections and $\mathbf{s}$ over the associated braid reflections.

An important and well-studied special case occurs if $W$ is actually a real reflection group, that is a Coxeter group, in which case the cyclotomic Hecke algebra becomes the well-known Iwahori-Hecke algebra of $W$. In this situation, all of the questions mentioned later in the paper were settled quite a while ago, so here we will be concerned exclusively with the non-real groups.

Bessis $[\mathbf{3}, 0.1(\mathrm{e})]$ has shown that $B(W)$ has a presentation of the form

$$
\left\langle\mathbf{s}_{1}, \ldots, \mathbf{s}_{n} \mid p_{j}\left(\mathbf{s}_{1}, \ldots, \mathbf{s}_{n}\right)=q_{j}\left(\mathbf{s}_{1}, \ldots, \mathbf{s}_{n}\right)\right\rangle,
$$

Received 22 September 2009.

2000 Mathematics Subject Classification 16Z05, $20 \mathrm{C08}$ (primary), 16A65 (secondary). 
where $\mathbf{s}_{i}$ are braid reflections whose images in $W$ form a minimal system of reflections needed to generate $W$ (thus if $W$ is irreducible we have $n=\operatorname{dim} V$ or $n=\operatorname{dim} V+1$ ) and where $\left(p_{j}, q_{j}\right)$ run over a finite set of pairs of positive words of equal length in the $\mathbf{s}_{i}$. One obtains a presentation of $W$ by adding the relations $\mathbf{s}_{i}^{d_{s_{i}}}=1$ where $d_{s_{i}}$ is the order of the reflection $s_{i} \in W$, which is the image of $\mathbf{s}_{i}$ (cf. [3,0.1(f)]).

A consequence is that the cyclotomic Hecke algebra specializes to the group algebra of $W$ under the map $u_{s, j} \mapsto \exp \left(2 \pi i j / d_{s}\right)$.

Explicit presentations of the form (1.1) of $B(W)$ and hence of $\mathcal{H}(W, \mathbf{u})$ are known for all irreducible reflection groups; see Broué, Malle and Rouquier [9] and the references given there, Bessis and Michel [5] and Bessis [4, Theorem 0.6].

The properties of cyclotomic Hecke algebras have been studied because of their (conjectured) role in the representation theory of finite reductive groups. Nevertheless, several important questions remain open at present, or have been settled only for some of the irreducible reflection groups. We recall them in $\S 2$.

Apart from these structural problems, there are questions of a more computational nature which need to be settled. We would like to be able to write down an explicit $A$-basis of $\mathcal{H}(W, \mathbf{u})$, with known structure constants. Furthermore, we would like to know explicit models for all irreducible representations of $\mathcal{H}(W, \mathbf{u})$. Again, these two questions have been solved for the imprimitive reflection groups $[\mathbf{2}, \mathbf{1 6}]$. In the present paper, we solve these computational problems for the primitive irreducible reflection groups of dimension at most three, which only leaves the five groups $G_{29}, G_{31}, G_{32}, G_{33}$ and $G_{34}$ (in Shephard and Todd's notation for the irreducible reflection groups) to be considered.

It is easy to see that the reflection representation $V$ of $W$ can be realized over the field $K_{W}$ generated by the traces of the elements of $W$ on $V$. It is a theorem of Benard and Bessis that all representations of $W$ can be realized over $K_{W}$.

Let $\mathcal{O}$ be the ring of integers of $K_{W}$ and let $\tilde{A}=\mathcal{O}\left[v_{s, i}, v_{s, i}^{-1}\right]_{s, i}$ where $v_{s, i}$ are such that $v_{s, i}^{e}=\exp \left(-2 \pi i j / d_{s}\right) u_{s, i}$, where $e$ is the order of the group of roots of unity in $K_{W}$. It has been shown in [14] that assuming Conjecture 2.2(a) below, the characters of $\mathcal{H}(W, \mathbf{u})$ take their values (on any basis of $\mathcal{H}(W, \mathbf{u})$ consisting of images of a subset of $B(W)$ ) in $\tilde{A}$. A consequence of our results here is that, whenever we can compute them, the representations of $\mathcal{H}(W, \mathbf{u})$ have a model where the matrices for the generators $\mathbf{s}_{i}$ have entries in the field generated by the corresponding character values.

\section{Some conjectures}

We start by recalling some basic conjectures on the structure and representation theory of cyclotomic Hecke algebras. The most basic conjecture states the following.

Conjecture 2.1. Let $W$ be a complex reflection group, $K=\operatorname{Frac}(A)$. Then:

(a) $\mathcal{H}(W, \mathbf{u}) \otimes_{A} K$ has dimension $|W|$;

(b) there exist pairwise non-isomorphic irreducible representations $\rho_{i}$ of $\mathcal{H}(W, \mathbf{u})$ over $\tilde{A}$ such that $\sum_{i} \operatorname{dim}\left(\rho_{i}\right)^{2}=|W|$.

Part (a) is known to hold for the infinite series by work of Ariki [1] and Broué and Malle [7], for the two-dimensional primitive groups by Etingof and Rains [11] and has been checked for the three-dimensional primitive groups by Müller (see Table 9). Our methods can prove part (b) in some cases, which shows that the dimension is at least that big, but we obtain no information on an upper bound. But, assuming these weak statements, we will derive the validity of an important stronger assertion. For this, now let $W$ be irreducible. Then it is known by $[\mathbf{4}$, Remark 12.4] that, except possibly for the case of $G_{31}$, the center of $B(W)$ is cyclic, generated 
by some element $\mathbf{z}$. We set $\boldsymbol{\pi}=\mathbf{z}^{|Z W|}$, with $Z W$ the center of $W$ (an element of the pure braid group $\left.\pi_{1}\left(V^{\text {reg }}, x_{0}\right)\right)$.

Conjecture 2.2. Let $W$ be an irreducible complex reflection group. Then:

(a) $\mathcal{H}(W, \mathbf{u})$ is free over $A$ of rank $|W|$;

(b) $\mathcal{H}(W, \mathbf{u})$ carries a non-degenerate symmetrizing form $t: \mathcal{H}(W, \mathbf{u}) \rightarrow A$ which makes it into a symmetric algebra, and such that

$$
t\left(T_{b^{-1}}\right)^{\vee}=t\left(T_{b \boldsymbol{\pi}}\right) / t\left(T_{\boldsymbol{\pi}}\right) \quad \text { for all } b \in B(W),
$$

where we denote by $b \mapsto T_{b}$ the natural map from $B(W) \rightarrow \mathcal{H}(W, \mathbf{u})$ and $x \mapsto x^{\vee}$ is the automorphism of $A$ given by $\mathbf{u} \mapsto \mathbf{u}^{-1}$.

Once Conjecture 2.2(a) has been established, it follows from Tits' deformation theorem that $\mathcal{H}(W, \mathbf{u})$ is a deformation of the group algebra of $W$, that is, it becomes isomorphic to the group algebra over a suitable finite extension of the field of fractions of $A$.

It was shown in $[8,2.1]$ that assuming part (a), there is at most one symmetrizing trace on $\mathcal{H}(W, \mathbf{u})$ satisfying part (b) which specializes to the canonical trace on $\mathbb{C} W$.

Given a split semi-simple symmetric algebra $H$ with a symmetrizing form $t$ such that $t(1)=1$, we define the Schur element $S_{\chi}$ attached to $\chi \in \operatorname{Irr}(H)$ by the property that

$$
t(x)=\sum_{\chi \in \operatorname{Irr}(H)} \chi(x) / S_{\chi} \quad \text { for all } x \in H .
$$

Let us denote by $\mathbf{s} \mapsto T_{\mathbf{s}}$ the natural map $B(W) \rightarrow \mathcal{H}(W, \mathbf{u})$. In $[\mathbf{1 3}, \mathbf{1 5}]$, assuming Conjecture 2.2(a) (which implies that $\mathcal{H}(W, \mathbf{u})$ is split semi-simple over a suitable extension of $A$ ), it was shown that for all exceptional complex reflection groups there is a unique symmetrizing trace such that $t\left(T_{x}\right)=0$ for $x \in E \backslash\{1\}$, where $E$ is a subset of $B(W)$ such that:

- all character values on $\left\{T_{x} \mid x \in E\right\}$ could be determined;

- equations (2.4) for $x \in E$ are sufficient in number to have a unique solution. For instance, it is enough that the image of $E$ in $W$ intersects all conjugacy classes.

Moreover, the corresponding Schur elements $S_{\chi}$ were determined in all cases. When specializing the Hecke algebra to the group algebra $\mathbb{C} W, t$ specializes to the canonical trace $t_{W}$ on $\mathbb{C} W$ given by $t_{W}(w)=\delta_{w, 1}$, so the Schur elements computed in [15] specialize to $|W| / \chi(1)$.

We fix this symmetrizing form $t$ described above. All of our computational verifications will depend on a suitable choice of basis for the cyclotomic Hecke algebra.

Lemma 2.5. Assume Conjecture 2.1. Let $C \subseteq \mathcal{H}(W, \mathbf{u})$ be of cardinality $|W|$ and specializing to $W \subseteq \mathbb{C} W$ under the specialization of $\mathcal{H}(W, \mathbf{u})$ to the group algebra. Then $C$ is a $K$-basis of $\mathcal{H}(W, \mathbf{u}) \otimes_{A} K$.

Proof. Indeed, by (2.4) the matrix $M:=t(x y)_{x, y \in C}$ has entries in the localization of $\tilde{A}$ at the collection of the Schur elements. Since the specialization of Schur elements is non-zero, we may specialize $M$ to obtain the corresponding matrix $M:=t_{W}(v w)_{v, w \in W}$ for $W$, which is a permutation matrix. Thus, $\operatorname{det}(M)$ is non-zero, and hence $C$ is $K$-linear independent. The claim then follows from Conjecture 2.1.

An obvious way to construct a set $C$ as above is by lifting the elements of $W$ to $B(W)$. We are looking for lifts which satisfy an additional property with respect to $t$.

Conjecture 2.6. There exists a section $W \rightarrow \mathbf{W}, w \mapsto \mathbf{w}$, of $W$ in $B(W)$ such that $\mathbf{W} \ni 1$, and such that for any $\mathbf{w} \in \mathbf{W} \backslash\{1\}$ we have $t\left(T_{\mathbf{w}}\right)=0$. 
According to Lemma $2.5,\left\{T_{\mathbf{w}} \mid w \in \mathbf{W}\right\}$ is a $K$-basis of $\mathcal{H}(W, \mathbf{u}) \otimes_{A} K$. Note, however, that in general it will not necessarily be an $A$-basis of $\mathcal{H}(W, \mathbf{u})$. Now (2.3) and Conjecture 2.6 are related as follows.

Proposition 2.7. Assume Conjecture 2.6. If either all irreducible representations of $\mathcal{H}(W, \mathbf{u})$ have models over $\tilde{A}$, or else $\left\{T_{\mathbf{w}} \mid w \in \mathbf{W}\right\}$ is an $A$-basis of $\mathcal{H}(W, \mathbf{u})$ then property $(2.3)$ is equivalent to:

$$
\text { for any } \mathbf{w} \in \mathbf{W}-\{1\} \text { we have } t\left(T_{\mathbf{w}^{-1} \boldsymbol{\pi}}\right)=0 \text {. }
$$

Proof. Let us extend $\vee$ to $\tilde{A}$ so that it does complex conjugation on $\mathcal{O}$ and sends $v_{i, j}$ to $v_{i, j}^{-1}$. Using equation (2.4) for $x=T_{b}^{-1}$ and $x=T_{b \boldsymbol{\pi}}$, condition (2.3) reads

$$
t\left(T_{\boldsymbol{\pi}}\right) \sum_{\chi \in \operatorname{Irr}(\mathcal{H}(W, \mathbf{u}))} \frac{\chi\left(T_{b}^{-1}\right)^{\vee}}{S_{\chi}^{\vee}}=\sum_{\chi \in \operatorname{Irr}(\mathcal{H}(W, \mathbf{u}))} \omega_{\chi}\left(T_{\boldsymbol{\pi}}\right) \frac{\chi\left(T_{b}\right)}{S_{\chi}},
$$

where $\omega_{\chi}$ is the central character of $\chi$. Under the standard specialization $\varphi: \tilde{A} \rightarrow \underline{\mathbb{C}, v_{s}, j} \mapsto 1$, we obviously have the following compatibility with complex conjugation: $\varphi\left(a^{\vee}\right)=\overline{\varphi(a)}$ for all $a \in \tilde{A}$. Thus

$$
\varphi\left(\chi\left(T_{b}^{-1}\right)^{\vee}\right)=\overline{\varphi\left(\chi\left(T_{b}^{-1}\right)\right)}=\overline{\chi\left(b^{-1}\right)}=\chi\left(b^{-1}\right)=\varphi\left(\chi\left(T_{b}\right)\right),
$$

whence $\chi\left(T_{b}^{-1}\right)^{\vee}=\chi\left(T_{b}\right)$ (note that by our assumptions all character values $\chi\left(T_{b}\right)$ lie in $\tilde{A}$ ). Our first equation then reads

$$
t\left(T_{\boldsymbol{\pi}}\right) \sum_{\chi \in \operatorname{Irr}(\mathcal{H}(W, \mathbf{u}))} \frac{\chi\left(T_{b}\right)}{S_{\chi}^{\vee}}=\sum_{\chi \in \operatorname{Irr}(\mathcal{H}(W, \mathbf{u}))} \omega_{\chi}\left(T_{\boldsymbol{\pi}}\right) \frac{\chi\left(T_{b}\right)}{S_{\chi}},
$$

which is a linear condition in $T_{b}$. Thus:

- if the equation holds for the image of $B(W)$ it holds for any element of $\mathcal{H}(W, \mathbf{u})$;

- it is sufficient to check the equation for a basis of $\mathcal{H}(W, \mathbf{u}) \otimes_{A} K$.

Note that $\left\{T_{\mathbf{w}}^{-1} \mid \mathbf{w} \in \mathbf{W}\right\}$ is still a basis of $\mathcal{H}(W, \mathbf{u}) \otimes_{A} K$ since it is the image of $\left\{T_{\mathbf{w}} \mid \mathbf{w} \in \mathbf{W}\right\}$ by the anti-automorphism $a_{1}$ of $[8,1.26]$. Writing the condition on this basis we get

$$
t\left(T_{\mathbf{w}}\right)^{\vee}=\frac{t\left(T_{\mathbf{w}^{-1} \boldsymbol{\pi}}\right)}{t\left(T_{\boldsymbol{\pi}}\right)} .
$$

This holds trivially for $\mathbf{w}=1$, and for the others $t\left(T_{\mathbf{w}}\right)^{\vee}=0$ whence the result.

Definition 2.9. We say that a section $\mathbf{W}$ is good if for any $\mathbf{w} \in \mathbf{W}-\{1\}$ we have $t\left(T_{\mathbf{w}}\right)=t\left(T_{\mathbf{w}^{-1} \boldsymbol{\pi}}\right)=0$, and the matrix $\left\{t\left(T_{\mathbf{w}_{\mathbf{w}^{\prime}}}\right)\right\}_{\mathbf{w}, \mathbf{w}^{\prime} \in \mathbf{W}}$ is in $\mathrm{GL}_{|W|}(A)$.

The notion of good section is the tool which will allow us to prove Conjecture 2.2 in quite a few cases, using the next proposition.

Proposition 2.10. Assume that $\mathbf{W}$ is a good section and that for a generating set $\mathbf{S}$ of $B(W)$ we have for all $\mathbf{s} \in \mathbf{S}, \mathbf{w}, \mathbf{w}^{\prime} \in \mathbf{W}$ that $t\left(T_{\mathbf{s w w}^{\prime}}\right) \in A$. Then $\mathcal{H}(W$, $\mathbf{u})$ satisfies Conjecture 2.2, and $\left\{T_{\mathbf{w}} \mid \mathbf{w} \in \mathbf{W}\right\}$ is an $A$-basis of $\mathcal{H}(W, \mathbf{u})$.

Proof. Let $M$ be the matrix $\left\{t\left(T_{\mathbf{w w}^{\prime}}\right)\right\}_{\mathbf{w}, \mathbf{w}^{\prime} \in \mathbf{W}}$. From the assumption $M \in \mathrm{GL}_{|W|}(A)$ it follows that the dual basis $\left\{T_{\mathbf{w}}^{\prime}\right\}$ of $\left\{T_{\mathbf{w}}\right\}$ with respect to $t$ lies in $A\left[T_{\mathbf{w}}\right]_{\mathbf{w} \in \mathbf{W}}$. It follows that $h \in \mathcal{H}(W, \mathbf{u})$ lies in $A\left[T_{\mathbf{w}}\right]_{\mathbf{w} \in \mathbf{W}}$ if and only if for any $\mathbf{w}$ we have $t\left(h T_{\mathbf{w}}\right) \in A$; indeed the coefficient of $h$ on $T_{\mathbf{w}}$ is $t\left(h T_{\mathbf{w}}^{\prime}\right)$ which is in $A$ if all the $t\left(h T_{\mathbf{w}}\right)$ are in $A$.

Thus the condition in the statement shows that $T_{\mathbf{s w}} \in A\left[T_{\mathbf{w}}\right]_{\mathbf{w} \in \mathbf{W}}$, that is that $T_{\mathbf{w}}$ is an $A$-basis. 


\section{Imprimitive groups}

Before turning to the main subject of the present paper, the exceptional complex reflection groups, we recall the current situation for the infinite series, that is, the imprimitive groups and the symmetric groups. Conjecture 2.2(a) has been verified in these cases by Ariki and Koike [2], Broué and Malle [7] and Ariki [1, 1.6(2)]. The properties of a symmetrizing form on $\mathcal{H}(G(d e, e, r), \mathbf{u})$ have been investigated by Malle and Mathas in [16]. It is not clear, though, that it satisfies the additional properties mentioned in Conjecture 2.2. Conjecture 2.6 has been verified for $G(d, 1, r)$ by Bremke and Malle [6].

Explicit models for the irreducible representations of the generic cyclotomic Hecke algebra for the imprimitive complex reflection group $G(d, 1, r)$ have been given by Ariki and Koike [2], and have been extended to $G(d e, e, r)$ by Ariki [1]. However, these models are over $K_{W}\left(u_{s, i}^{1 / e}\right)_{s, i}$.

Models for the case $G(d, 1, r)$ are known over $A$, using the fact that this is a cellular algebra, and that the generators act with coefficients in $A$ on a cellular basis. For example this can be seen from Dipper, James and Mathas [10]. Let the generators of $\mathcal{H}(W, \mathbf{u})$ correspond to the diagram

$$
\bigcirc_{T_{0}}=\bigcirc_{T_{1}}-\bigcirc_{T_{2}} \cdots \bigcirc_{T_{r-1}}^{\bigcirc}
$$

where $T_{0}$ has parameters $Q_{1}=u_{0,0}, \ldots, Q_{d}=u_{0, d-1}$ and the $T_{i}(i \neq 1)$ have parameters $q=u_{1,0},-1=u_{1,1}$. Then the action of the $T_{i}$ in a cellular basis is given by $[\mathbf{1 0}, 3.15$ and 3.18], while the action of $T_{0}$ is given by [10,3.20] (note that only the term $x_{1}$ of [10] subsists in the model for the representation $\lambda$ ).

We are not aware of similarly nice integral/rational models for the representations of $\mathcal{H}(G(d e, e, r), \mathbf{u})$, where $e>1$.

\section{Two-dimensional primitive groups}

In this section we describe the construction of models for the irreducible representations of the Hecke algebras $\mathcal{H}(W, \mathbf{u})$, where $W$ is a primitive two-dimensional reflection group, so one of the groups $G_{4}, \ldots, G_{22}$. We first describe several reductions.

Step 1. It is sufficient to find models in the case of $G_{7}, G_{11}$ and $G_{19}$.

The braid groups of $G_{7}, G_{11}$ and $G_{19}$ are isomorphic to the same group

$$
B:=\left\langle\mathbf{s}_{1}, \mathbf{s}_{2}, \mathbf{s}_{3} \mid \mathbf{s}_{1} \mathbf{s}_{2} \mathbf{s}_{3}=\mathbf{s}_{2} \mathbf{s}_{3} \mathbf{s}_{1}=\mathbf{s}_{3} \mathbf{s}_{1} \mathbf{s}_{2}\right\rangle
$$

(see $[\mathbf{9}, \S 5]$ ). Let $\mathbf{u}=\left(x_{1}, x_{2} ; y_{1} ; y_{2} ; y_{3} ; z_{1}, \ldots, z_{k}\right)$, where $k=3$ for $G_{7}$ (respectively 4,5 for $\left.G_{11}, G_{19}\right)$. The cyclotomic Hecke algebra $\mathcal{H}(W, \mathbf{u})$ of $G_{7}$ (respectively $G_{11}, G_{19}$ ) is the quotient of the group algebra of $B$ over $\mathbb{Z}\left[\mathbf{u}, \mathbf{u}^{-1}\right]$ by the relations

$$
\left(\mathbf{s}_{1}-x_{1}\right)\left(\mathbf{s}_{1}-x_{2}\right)=0, \quad\left(\mathbf{s}_{2}-y_{1}\right)\left(\mathbf{s}_{2}-y_{2}\right)\left(\mathbf{s}_{2}-y_{3}\right)=0, \quad \prod_{i=1}^{i=k}\left(\mathbf{s}_{3}-z_{i}\right)=0 .
$$

In turn the Hecke algebras for $G_{4}$ to $G_{6}$ are subalgebras of suitable partial specializations of that for $G_{7}$ (the same holds for $G_{8}$ to $G_{15}$ with respect to $G_{11}$ and for $G_{16}$ to $G_{22}$ with respect to $G_{19}$ ) (see [13, Proposition 4.2]). More precisely, in each case, these algebras are generated by suitable conjugates of a subset of the generators (or of some power of them), while the other generators are specialized to the group algebra. The necessary generators are collected in Table 1.

Moreover, each irreducible representation of the Hecke algebra of any of the groups $G_{4}, \ldots, G_{22}$ can be obtained as the restriction of an irreducible representation of the Hecke algebra of one of $G_{7}, G_{11}$ or $G_{19}$. It follows that it is sufficient to determine the representations 
of the Hecke algebras of $G_{7}, G_{11}$ and $G_{19}$ to determine the representations of the Hecke algebras of all two-dimensional primitive reflection groups.

Step 2. It is sufficient to compute irreducible representations of $B$ of dimension $2 \leqslant d \leqslant 6$, with an additional condition on the eigenvalues of the generators.

The irreducible representations of $G_{7}$ have dimension one, two or three, those of $G_{11}$ dimension one to four and those of $G_{19}$ dimension one to six. It follows that any two-dimensional representation of $B$ gives a representation of $\mathcal{H}(W, \mathbf{u})$ where $W$ is any of $G_{7}, G_{11}, G_{19}$; any three-dimensional representation of $B$ where $\mathbf{s}_{1}$ has only two distinct eigenvalues gives a representation of the same algebras; any four-dimensional representation of $B$ where $\mathbf{s}_{1}$ has only two distinct eigenvalues and $\mathbf{s}_{2}$ has only three distinct eigenvalues gives a representation of $\mathcal{H}(W, \mathbf{u})$ where $W$ is any of $G_{11}, G_{19}$; finally any five- or six-dimensional representation of $B$ where $\mathbf{s}_{1}$ has only two distinct eigenvalues, $\mathbf{s}_{2}$ has only three distinct eigenvalues and $\mathbf{s}_{3}$ has only five distinct eigenvalues gives a representation of $\mathcal{H}\left(G_{19}, \mathbf{u}\right)$.

Step 3. It is sufficient to compute one irreducible representations of $B$ in each dimension $2 \leqslant d \leqslant 6$.

For each dimension (from one to six) and each $W \in\left\{G_{7}, G_{11}, G_{19}\right\}$, the irreducible representations of $\mathcal{H}(W, \mathbf{u})$ (up to isomorphism) form a single orbit under the Galois automorphisms corresponding to permuting the $x_{i}$, the $y_{i}$, the $z_{i}$ among themselves. It transpires that we just need to find one representation of $B$ of the right dimension with the required number of eigenvalues.

It turns out that one can find such representations of $B$ by matrices of the form

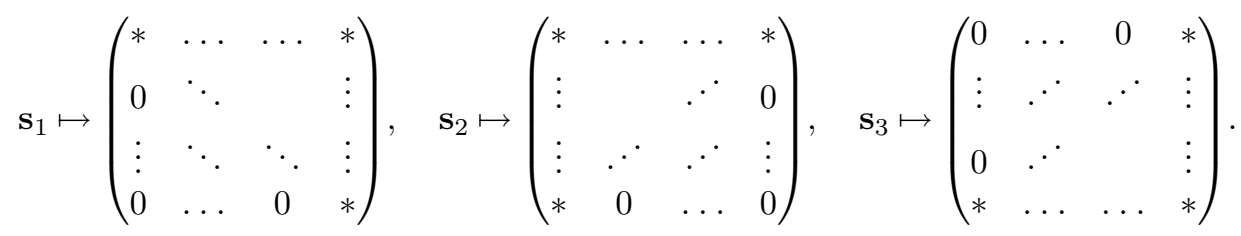

A solution for the two-dimensional representation is

$$
\mathbf{s}_{1} \mapsto\left(\begin{array}{cc}
x_{1} & \frac{y_{1}+y_{2}}{y_{1} y_{2}}-\frac{\left(z_{1}+z_{2}\right) x_{2}}{r} \\
0 & x_{2}
\end{array}\right), \quad \mathbf{s}_{2} \mapsto\left(\begin{array}{cc}
y_{1}+y_{2} & 1 / x_{1} \\
-y_{1} y_{2} x_{1} & 0
\end{array}\right), \quad \mathbf{s}_{3} \mapsto\left(\begin{array}{cc}
0 & \frac{-r}{y_{1} y_{2} x_{1} x_{2}} \\
r & z_{1}+z_{2}
\end{array}\right),
$$

TABLE 1. Generators for Hecke algebras of two-dimensional primitive groups.

\begin{tabular}{cc}
\hline$W$ & Generators of $\mathcal{H}(W)$ \\
\hline$G_{4}, G_{8}, G_{16}$ & $\mathbf{s}_{3},{ }^{\mathbf{s}_{1}} \mathbf{s}_{3}$ \\
$G_{5}, G_{10}, G_{18}$ & $\mathbf{s}_{2}, \mathbf{s}_{3}$ \\
$G_{6}, G_{9}, G_{17}$ & $\mathbf{s}_{1}, \mathbf{s}_{3}$ \\
$G_{14}, G_{21}$ & $\mathbf{s}_{1}, \mathbf{s}_{2}$ \\
$G_{12}, G_{22}$ & $\mathbf{s}_{1},{ }^{\mathbf{s}_{2}} \mathbf{s}_{1}, \mathbf{s}_{1}$ \\
$G_{20}$ & $\mathbf{s}_{2}, \mathbf{s}_{1} \mathbf{s}_{2}$ \\
$G_{13}$ & $\mathbf{s}_{3}^{2}, \mathbf{s}_{1}, \mathbf{s}_{1}^{\mathbf{s}_{2}}$ \\
$G_{15}$ & $\mathbf{s}_{1}, \mathbf{s}_{2}, \mathbf{s}_{3}^{2}$ \\
\hline
\end{tabular}


where $r=\sqrt{x_{1} x_{2} y_{1} y_{2} z_{1} z_{2}}$. Note that the irrationality $r$ occurring in the matrices is necessary [14, Table 8.1].

A solution for the three-dimensional representation is

$$
\begin{gathered}
\left.\mathbf{s}_{1} \mapsto \begin{array}{ccc}
x_{1} & 0 & \left(\left(z_{2} z_{3}+z_{1} z_{3}+z_{1} z_{2}\right) x_{2} x_{1} r^{-1}-\frac{\left(y_{3}+y_{1}+y_{2}\right) r}{y_{1} y_{2} y_{3}}\right) z_{1}^{-1} \\
0 & x_{1} & -r\left(y_{1} y_{2} y_{3} z_{1}\right)^{-1} \\
0 & 0 & x_{2}
\end{array}\right), \\
\mathbf{s}_{2} \mapsto\left(\begin{array}{ccc}
y_{1}+y_{2}+y_{3}-r\left(x_{1} z_{1}\right)^{-1} & a z_{1}-1 & -1 \\
1 & r\left(x_{1} z_{1}\right)^{-1} & 0 \\
y_{1} y_{2} y_{3} x_{1} z_{1} r^{-1} & 0 & 0
\end{array}\right) \\
\mathbf{s}_{3} \mapsto\left(\begin{array}{ccc}
0 & 0 & z_{2} z_{3} x_{1} r^{-1} \\
0 & z_{1} & 0 \\
-r x_{1}{ }^{-1} & a & z_{3}+z_{2}
\end{array}\right)
\end{gathered}
$$

where

$$
a=\left(y_{3}+y_{1}+y_{2}\right) r x_{1}^{-1}-\left(y_{1} y_{3}+y_{1} y_{2}+y_{3} y_{2}\right) z_{1}+y_{1} y_{2} y_{3}\left(x_{1} z_{1}^{2}-x_{2} z_{2} z_{3}\right) r^{-1}
$$

and where $r=\sqrt[3]{x_{1}^{2} x_{2} y_{1} y_{2} y_{3} z_{1} z_{2} z_{3}}$.

A solution for the four-dimensional representation is

$$
\begin{aligned}
& \mathbf{s}_{1} \mapsto\left(\begin{array}{cccc}
x_{1} & 0 & x_{1} a-x_{1} x_{2} y_{1} \frac{b}{r} & x_{1}\left(1+\frac{y_{1}}{y_{3}}\right)-\frac{r}{y_{3}} \sum_{i} \frac{1}{z_{i}} \\
0 & x_{1} & \frac{1}{y_{1}}+\frac{1}{y_{2}} & -\frac{x_{2}}{r^{3}} \\
0 & 0 & x_{2} & 0 \\
0 & 0 & 0 & x_{2}
\end{array}\right), \\
& \mathbf{s}_{2} \mapsto\left(\begin{array}{cccc}
y_{3}+y_{1} & x_{1} y_{1} y_{2} a & y_{1} a & y_{1} \\
0 & y_{1}+y_{2} & 1 / x_{1} & 0 \\
0 & -x_{1} y_{1} y_{2} & 0 & 0 \\
-y_{3} & 0 & 0 & 0
\end{array}\right) \\
& \mathbf{s}_{3} \mapsto\left(\begin{array}{cccc}
0 & 0 & 0 & -r /\left(y_{3} x_{2}\right) \\
0 & 0 & -r /\left(y_{2} x_{1} x_{2} y_{1}\right) & 0 \\
0 & r & 0 & 1 / r^{2} \\
r /\left(x_{1} y_{1}\right) & -r a & b & \sum_{i} z_{i}
\end{array}\right),
\end{aligned}
$$

where

$$
a=x_{1} x_{2} y_{1} y_{2} \prod_{j} z_{j}\left(\sum_{i} \frac{1}{z_{i}}\right)-r^{2} \sum_{i=1}^{4} z_{i}, \quad b=x_{1} x_{2} y_{1}\left(y_{2}+y_{3}\right) \prod_{i} z_{i}-r^{2} \sum_{i<j} z_{i} z_{j}
$$

and where $r=\sqrt[4]{x_{1}^{2} x_{2}^{2} y_{1} y_{2} y_{3}^{2} z_{1} z_{2} z_{3} z_{4}}$.

We refer to the GAP-part of the Chevie system [17] for solutions for the five-dimensional and six-dimensional representations of $B$. By our above reductions, this completes the construction of the irreducible representations of all cyclotomic Hecke algebras attached to two-dimensional exceptional complex reflection groups. 


\section{Hensel lifting and Padé approximation}

We now describe computational techniques used to obtain models for irreducible representations of Hecke algebras for higher-dimensional primitive complex reflection groups. It is not an algorithm in the sense that it does not always succeed, but in the case of oneparameter algebras, it turned out to have a good rate of success. It consists of Hensel lifting representations of $W$ to $\mathcal{H}(W)$, combined with Padé approximation.

We note that for groups generated by true reflections which are all conjugate, such as $G_{24}$, $G_{27}, G_{29}, G_{31}, G_{33}$ and $G_{34}$, there are only two parameters $u_{s, 0}$ and $u_{s, 1}$, and with the usual normalization $u_{s, 1}=-1$ (corresponding to replacing $\mathbf{s}$ by $-\mathbf{s} / u_{s, 1}$ ) there is only one parameter $q=-u_{s, 0} / u_{s, 1}$. We will write $\mathcal{H}(W, q)$ for such an algebra.

\subsection{Hensel lifting representations of $W$}

We start with a presentation of $B(W)$, of the form

$$
\left\langle\mathbf{s}_{1}, \ldots, \mathbf{s}_{n} \mid p_{j}\left(\mathbf{s}_{1}, \ldots, \mathbf{s}_{n}\right)=q_{j}\left(\mathbf{s}_{1}, \ldots, \mathbf{s}_{n}\right)\right\rangle
$$

as explained in (1.1).

If $\rho_{q}: \mathcal{H}(W, q) \rightarrow M_{l \times l}(\mathbb{C}(q))$ is a representation of $\mathcal{H}(W, q)$ over $\mathbb{C}(q)$, and if $M_{i}=\rho_{q}\left(\mathbf{s}_{i}\right)$, the idea consists of writing $M_{i}$ as a series in the variable $r:=q-1$. If we have such an expansion $M_{i}=M_{i}^{(0)}+r M_{i}^{(1)}+r^{2} M_{i}^{(2)}+\ldots$ where $M_{i}^{(j)} \in M_{l \times l}(\mathbb{C})$, then $M_{i}^{(0)}$ is the specialization $\rho_{1}$ of $\rho_{q}$ at $q=1$, a representation of $W$.

Conversely, if we start with a representation $\rho_{1}$ of $W$, we may try to extend it to a representation of $\mathcal{H}(W, q)$ by solving the system of equations

$$
\left(M_{i}+1\right)\left(M_{i}-r-1\right)=0 \quad \text { and } \quad p_{j}\left(M_{1}, \ldots, M_{n}\right)=q_{j}\left(M_{1}, \ldots, M_{n}\right),
$$

where $M_{i}=M_{i}^{(0)}+r M_{i}^{(1)}+r^{2} M_{i}^{(2)}+\ldots \in M_{l \times l}(\mathbb{C}((r)))$ are formal power series with $M_{i}^{(0)}=$ $\rho_{1}\left(\mathbf{s}_{i}\right)$. The point here is that if we already have a solution $M_{i}^{(j)}$ for $j<j_{0}$ (where $j_{0} \geqslant 1$ ) then the equations for $M_{i}^{\left(j_{0}\right)}$ form a system of linear equations, which, if $v$ is the vector of all the entries $\left(M_{i}^{\left(j_{0}\right)}\right)_{k, l}$ of the matrices $M_{i}^{\left(j_{0}\right)}$, has the form $\Lambda v=N_{j_{0}}$, for a matrix $\Lambda$ which is independent of $j_{0}$.

Unfortunately the matrix $\Lambda$ does not have full rank in practice. To try to solve the above system, we choose for $j_{0}=1$ a matrix $\Lambda^{\prime}$ of full rank extending $\Lambda$, and then solve iteratively each step $j_{0}$ by setting $v=\Lambda^{\prime-1} N_{j_{0}}$. We thus get a representation of $\mathcal{H}(W, q)$ with coefficients in $\mathbb{C}[[r]]$; actually in $K[[r]]$ if $K$ is the field where the entries of the matrices $M_{i}^{(0)}$ lie.

In our computations it happened quite often that this representation is actually over $K(r)=K(q)$. This is the point of the method. To increase the probability that this happens, we found a number of heuristics:

- as equations added to $\Lambda$ to make $\Lambda^{\prime}$, we first try to add equations specifying that undetermined entries in $M_{i}^{(1)}$ where the corresponding entry in $M_{i}^{(0)}$ is 0 should also be 0 ;

- if the chosen model of $\rho_{1}$ given by the matrices $M_{i}^{(0)}$ does not give good results, change the model randomly (but such that it is still 'simple') until a better result occurs.

\subsection{Recognizing the entries}

To recognize that the obtained series $M_{i} \in M_{l \times l}(K[[r]])$ lies in $K(r)$, we use Padé approximation: if a series $h \in K[[r]]$, which can be assumed to have a non-zero constant coefficient, is the expansion of $f / g \in K(r)$ where $f, g \in K[r]$ are of degree less than $d$ and $g(0)=1$, then $f$ and $g$ are determined by solving linear equations involving only the first $2 d$ terms of $h$. If these linear equations have a solution, we say that $f / g$ is a Padé approximant of $h$. 
This is applied to the (approximate) entries of $M_{i}$ as follows: we compute Padé approximants for increasing $d$, until they become stationary, which generally means that we have found a solution in $K(r)$.

Note that it is very easy afterwards to check whether the result of our computations does indeed define a representation of $\mathcal{H}(W, q)$, by just evaluating the relations of $\mathcal{H}(W, q)$.

The representations of $\mathcal{H}(W, q)$ are in general not defined over $\mathbb{C}(q)$ but over $\mathbb{C}\left(q^{1 / e}\right)$ where $e$ is the order of the group of roots of unity in $K_{W}$. To handle this case it is sufficient to take $r:=q^{1 / e}-1$ as a variable and apply the same construction.

\subsection{Finding good models for representations of $W$}

To start the process we needed to get a complete set of models for the irreducible representations of $W$. For this, we used the following techniques:

- get new representations from known representations by tensoring by linear characters and applying Galois actions;

- get new representations as Schur functors of known representations (when such Schur functors happen to give an irreducible representation; an example is that the exterior powers $\Lambda^{i} V$ are always irreducible and the symmetric square $S^{2} V$ is irreducible if $W$ is not real). We have written a Chevie-program to compute general Schur functors to do this.

For example only seven of the 90 representations of dimension at most 60 of $G_{32}$ cannot be obtained by the above process starting from the reflection representation. To get the remaining representations, we need one more technique:

- obtain the desired representation as a component of multiplicity 1 in the tensor product of two known representations. Then compute a model by explicitly computing the projector on the desired isotypic component.

It turns out that all irreducible representations of exceptional complex reflection groups can be obtained from the reflection representation applying these three steps. To compute the projector on the isotypic component, we explicitly compute the image of the class sums of $W$ in the representation, by enumerating the elements of $W$ as words in the generators and computing their images. We have carried out this computation for all groups considered except $G_{34}$ where this would need to add together billions of matrices of rank several tens of thousands, which is a larger computation than those we have attempted.

For the questions to be considered below, but also for other computational purposes, it is desirable to have a model with few non-zero entries, which are integral if possible. We try to achieve this by performing suitable base changes on the first model. A good heuristic which tends to simplify the model a lot is to use a basis consisting of one-dimensional intersections of eigenspaces of the matrices $M_{i}^{(0)}$.

An example of a representation obtained by the methods of this section and that we could not obtain in another way is the representation $\phi_{8,5}$ of $\mathcal{H}\left(G_{24},\{x, y\}\right)$ (here $v=\sqrt{-x y}$, and $\mathbf{s}, \mathbf{t}, \mathbf{u}$ are the generators in the presentation $P_{1}$ given in $\left.\S 6.1\right)$ :

$$
\mathbf{s} \mapsto\left(\begin{array}{cccccccc}
\cdot & \cdot & \cdot & \cdot & \cdot & \cdot & \cdot & -x \\
\cdot & x+y & \cdot & \cdot & y & \cdot & \cdot & \cdot \\
\cdot & \cdot & x & -v y+x y & \cdot & \cdot & -x^{2} & \cdot \\
\cdot & \cdot & \cdot & y & \cdot & \cdot & \cdot & \cdot \\
\cdot & -x & \cdot & \cdot & \cdot & \cdot & \cdot & \cdot \\
\cdot & \cdot & \cdot & x & \cdot & x & -v-y & \cdot \\
\cdot & \cdot & \cdot & \cdot & \cdot & \cdot & y & \cdot \\
y & \cdot & \cdot & \cdot & \cdot & \cdot & \cdot & x+y
\end{array}\right)
$$




$$
\begin{gathered}
\mathbf{t} \mapsto\left(\begin{array}{cccccccc}
x & \cdot & \cdot & v & \cdot & \cdot & \cdot & -y \\
\cdot & x & \cdot & v & x & \cdot & \cdot & \cdot \\
\cdot & \cdot & x+y & \cdot & \cdot & \cdot & -x y & \cdot \\
\cdot & \cdot & \cdot & y & \cdot & \cdot & \cdot & \cdot \\
\cdot & \cdot & \cdot & \cdot & y & \cdot & \cdot & \cdot \\
\cdot & \cdot & -1 & x & -v & x & x & v \\
\cdot & \cdot & 1 & \cdot & \cdot & \cdot & \cdot & \cdot \\
\cdot & \cdot & \cdot & \cdot & \cdot & \cdot & \cdot & y
\end{array}\right), \\
\mathbf{u} \mapsto\left(\begin{array}{cccccccc}
y & \cdot & \cdot & \cdot & \cdot & \cdot & \cdot & \cdot \\
\cdot & x & \cdot & \cdot & x & \cdot & -v & \cdot \\
-x y & \cdot & x & \cdot & -v y & v y & v y-x y-x^{2} & \cdot \\
\cdot & \cdot & \cdot & x & \cdot & -y & -v-y & \cdot \\
\cdot & \cdot & \cdot & \cdot & y & \cdot & \cdot & \cdot \\
\cdot & \cdot & \cdot & \cdot & \cdot & y & & \cdot \\
\cdot & \cdot & \cdot & \cdot & \cdot & \cdot & & \cdot \\
x & \cdot & \cdot & \cdot & \cdot & \cdot & x & \cdot
\end{array}\right) .
\end{gathered}
$$

Another example is the representation $\phi_{8,6}$ of $\mathcal{H}\left(G_{27},\{x, y\}\right)$ where again $v=\sqrt{-x y}$, and $\mathbf{s}, \mathbf{t}, \mathbf{u}$ are the generators in the presentation $P_{1}$ given in $\S 6.2$ :

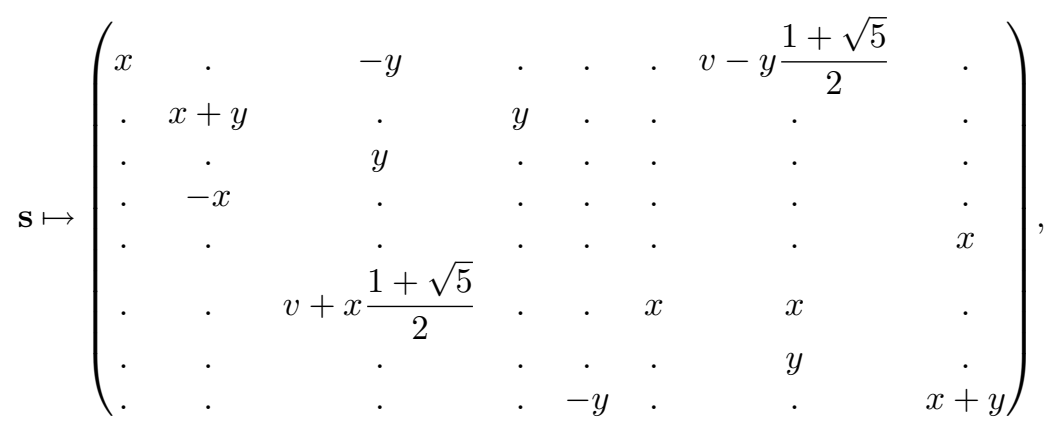

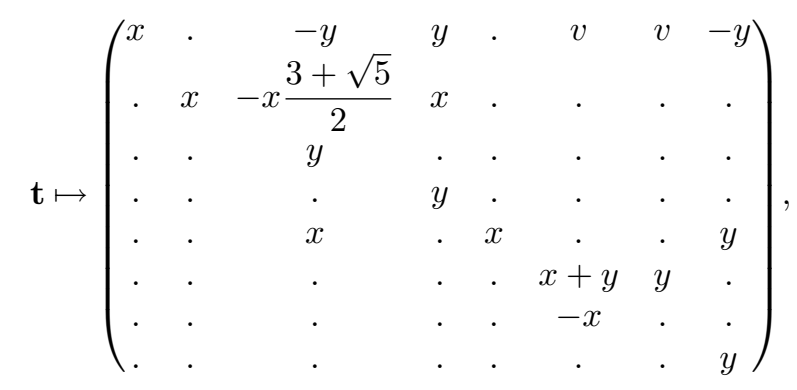

$$
\begin{aligned}
& \mathbf{u} \mapsto\left(\begin{array}{cccccccc}
\cdot & \cdot & -x & \cdot & \cdot & \cdot & \cdot & \cdot \\
\cdot & x & \cdot & x & \cdot & \cdot & -y \frac{3+\sqrt{5}}{2} & \cdot \\
y & \cdot & x+y & \cdot & \cdot & \cdot & \cdot & \cdot \\
\cdot & \cdot & \cdot & y & \cdot & \cdot & \cdot & \cdot \\
\cdot & \cdot & \cdot & \cdot & y & \cdot & \cdot & \cdot \\
v & \cdot & v & -y & v & x & x & \cdot \\
\cdot & \cdot & \cdot & \cdot & \cdot & \cdot & y & \cdot \\
\cdot & \cdot & \cdot & \cdot & -x & \cdot & v & x
\end{array}\right) .
\end{aligned}
$$




\section{Presentations of $B\left(G_{24}\right)$ to $B\left(G_{34}\right)$}

In [5] we considered presentations of exceptional complex braid groups, and proposed several presentations for $B\left(G_{24}\right), B\left(G_{27}\right), B\left(G_{29}\right), B\left(G_{33}\right)$ and $B\left(G_{34}\right)$. In the context of our current work, it will be important to consider alternative presentations, since at least two of the properties we consider (the existence of $W$-graphs and the vanishing of the trace on minimal length elements) turn out to depend on the presentation, with each time a presentation faring better than the others with respect to these properties. We have noticed a framework in which these various presentations fit and can be systematically recovered.

Since the groups above are well-generated, they have a unique maximal reflection degree $h$ called the Coxeter number of $W$. We proved in [5] that in each case, the product $\delta=\mathbf{s}_{1} \ldots \mathbf{s}_{n}$ of the generators of the braid group in a certain order is an $h$ th root of the generator of the center of the pure braid group. The image $c$ of $\delta$ in $W$ is $e^{2 i \pi / h}$-regular in the sense of Springer, and if choosing for the basepoint a $e^{2 i \pi / h}$-regular eigenvector $x$ of $c$, the element $\delta$ corresponds to a path joining $x$ to $e^{2 i \pi / h} x$ (these two points coincide in $V^{\text {reg }} / W$ ).

We consider the Hurwitz action of the ordinary Artin braid group $B_{n}$, the group with presentation

$$
\left.\left\langle\sigma_{1}, \ldots, \sigma_{n-1}\right| \sigma_{i} \sigma_{j}=\sigma_{j} \sigma_{i} \text { if }|i-j|>1, \sigma_{i} \sigma_{i+1} \sigma_{i}=\sigma_{i+1} \sigma_{i} \sigma_{i+1}\right\rangle
$$

on decompositions $\delta=\mathbf{s}_{1} \ldots \mathbf{s}_{n}$ of $\delta$, given by

$$
\sigma_{i}:\left(\mathbf{s}_{1}, \ldots, \mathbf{s}_{n}\right) \mapsto\left(\mathbf{s}_{1}, \ldots, \mathbf{s}_{i+1}, \mathbf{s}_{i}^{\mathbf{s}_{i+1}}, \ldots, \mathbf{s}_{n}\right),
$$

so that

$$
\sigma_{i}^{-1}:\left(\mathbf{s}_{1}, \ldots, \mathbf{s}_{n}\right) \mapsto\left(\mathbf{s}_{1}, \ldots, \mathbf{s}_{i} \mathbf{s}_{i+1}, \mathbf{s}_{i}, \ldots, \mathbf{s}_{n}\right) .
$$

We thus obtain new decompositions of $\delta$ into a product of $n$ braid reflections.

Bessis has shown in [4] that the orbit of the Hurwitz action on decompositions of $\delta$ is finite, of cardinality $n ! h^{n} /|W|$. What we noticed is that all the presentations of [5] correspond to taking as a set of generators the ones which appear in one decomposition in the Hurwitz orbit; in the case of $G_{24}$ and $G_{27}$ any element of a Hurwitz orbit corresponds up to some permutation to one of the presentations given in [5]; in the other cases some other presentations may appear.

We now give the results in each case. We found that the 'quality' of a presentation seems to be correlated to how 'spread out' their 'Poincaré' polynomial $\sum_{w \in W} q^{l(w)}$ is (where $l(w)$ is the minimal length in terms of the generators needed to write $w$ ); the presentations where the Poincaré polynomial has a higher degree are better.

\subsection{Presentations for $B\left(G_{24}\right)$}

A Hurwitz orbit of $\delta$ has 49 elements. Three different presentations appear along an orbit.

$P_{1}$. The presentation $P_{1}$ is

$$
\left\langle\mathbf{s}, \mathbf{t}, \mathbf{u} \mid \mathbf{s t s}=\mathbf{t s t}, \mathbf{t u t u}=\mathbf{u t u t}, \mathbf{s u s}=\mathbf{u s u},(\mathbf{t u s})^{3}=\mathbf{u t u}(\mathbf{s t u})^{2}\right\rangle .
$$

It appears 21 times in a Hurwitz orbit. Its Poincaré polynomial is

$$
\begin{aligned}
& q^{15}+3 q^{14}+6 q^{13}+12 q^{12}+27 q^{11}+46 q^{10}+55 q^{9}+54 q^{8}+44 q^{7} \\
& \quad+31 q^{6}+22 q^{5}+15 q^{4}+10 q^{3}+6 q^{2}+3 q+1 .
\end{aligned}
$$

$P_{2}$. The presentation $P_{2}$ is

$$
\left\langle\mathbf{s}, \mathbf{t}, \mathbf{u} \mid \mathbf{s t s t}=\mathbf{t s t s}, \mathbf{t u t u}=\mathbf{u t u t}, \mathbf{s u s}=\mathbf{u s u}, \mathbf{t}(\mathbf{s t u})^{2}=(\mathbf{s t u})^{2} \mathbf{s}\right\rangle .
$$


We get $P_{2}$ from $P_{1}$ by taking $\left\{\mathbf{s}, \mathbf{t u t}^{-1}, \mathbf{t}\right\}$ as generators. It appears 21 times in a Hurwitz orbit. Its Poincaré polynomial is

$q^{13}+4 q^{12}+16 q^{11}+39 q^{10}+56 q^{9}+58 q^{8}+52 q^{7}+42 q^{6}+29 q^{5}+18 q^{4}+11 q^{3}+6 q^{2}+3 q+1$.

$P_{3}$. The presentation $P_{3}$ is

$\left\langle\mathbf{s}, \mathbf{t}, \mathbf{u} \mid \mathbf{s t s t}=\mathbf{t s t s}, \mathbf{t u t u}=\mathbf{u t u t}, \mathbf{s u s u}=\mathbf{u s u s},(\mathbf{t u s})^{2} \mathbf{t}=(\mathbf{s t u})^{2} \mathbf{s}=(\mathbf{u s t})^{2} \mathbf{u}\right\rangle$.

We get $P_{3}$ from $P_{1}$ by taking $\left\{\mathbf{t}, \mathbf{u}, \mathbf{u}^{-1} \mathbf{t}^{-1} \mathbf{s t u}\right\}$ as generators. It appears seven times in a Hurwitz orbit. Its Poincaré polynomial is

$$
q^{13}+5 q^{12}+12 q^{11}+24 q^{10}+45 q^{9}+54 q^{8}+59 q^{7}+57 q^{6}+36 q^{5}+21 q^{4}+12 q^{3}+6 q^{2}+3 q+1 \text {. }
$$

\subsection{Presentations for $B\left(G_{27}\right)$}

A Hurwitz orbit of $\delta$ has 75 elements. Five different presentations appear along an orbit, each fifteen times.

$P_{1}$. The presentation $P_{1}$ is

$$
\left.\langle\mathbf{s}, \mathbf{t}, \mathbf{u}| \mathbf{t s t}=\mathbf{s t s}, \mathbf{u s u}=\mathbf{s u s}, \mathbf{u t u t}=\mathbf{t u t u}, \mathbf{u t u}(\mathbf{s t u})^{3}=(\mathbf{t u s})^{3} \text { tut }\right\rangle .
$$

Its Poincaré polynomial is

$$
\begin{aligned}
& q^{25}+5 q^{24}+12 q^{23}+26 q^{22}+51 q^{21}+88 q^{20}+125 q^{19}+150 q^{18}+168 q^{17}+191 q^{16} \\
& \quad+218 q^{15}+223 q^{14}+200 q^{13}+168 q^{12}+139 q^{11}+114 q^{10}+87 q^{9}+62 q^{8} \\
& \quad+44 q^{7}+31 q^{6}+22 q^{5}+15 q^{4}+10 q^{3}+6 q^{2}+3 q+1 .
\end{aligned}
$$

$P_{2}$. The presentation $P_{2}$ is

$$
\left.\langle\mathbf{s}, \mathbf{t}, \mathbf{u}| \mathbf{s u s}=\mathbf{u s u}, \mathbf{s t s t}=\mathbf{t s t s}, \text { tutut }=\mathbf{u t u t u},(\mathbf{u t s})^{2} \mathbf{t}=\mathbf{s}(\mathbf{u t s})^{2}\right\rangle .
$$

We get $P_{2}$ from $P_{1}$ by taking $\left\{\mathbf{t}, \mathbf{t} \mathbf{u t} \mathbf{t}^{-1}, \mathbf{s}\right\}$ as generators. Its Poincaré polynomial is

$$
\begin{aligned}
& q^{21}+6 q^{20}+22 q^{19}+59 q^{18}+107 q^{17}+152 q^{16}+208 q^{15}+256 q^{14}+270 q^{13} \\
& \quad+255 q^{12}+218 q^{11}+177 q^{10}+137 q^{9}+100 q^{8}+71 q^{7}+49 q^{6}+32 q^{5} \\
& \quad+19 q^{4}+11 q^{3}+6 q^{2}+3 q+1
\end{aligned}
$$

$P_{3}$. The presentation $P_{3}$ is

$$
\left.\langle\mathbf{s}, \mathbf{t}, \mathbf{u}| \mathbf{s t s}=\mathbf{t s t}, \text { tutut }=\mathbf{u t u t u}, \mathbf{s u s}=\mathbf{u s u}, \mathbf{t u t u}(\mathbf{s t u})^{2}=\mathbf{u}(\mathbf{t u s})^{3}\right\rangle .
$$

We get $P_{3}$ from $P_{1}$ by taking $\left\{\mathbf{s}, \mathbf{s}^{-1} \mathbf{u s}, \mathbf{t}\right\}$ as generators. Its Poincaré polynomial is

$$
\begin{aligned}
& q^{23}+3 q^{22}+6 q^{21}+21 q^{20}+60 q^{19}+121 q^{18}+164 q^{17}+192 q^{16}+228 q^{15} \\
& \quad+256 q^{14}+245 q^{13}+210 q^{12}+175 q^{11}+138 q^{10}+106 q^{9}+78 q^{8} \\
& \quad+57 q^{7}+38 q^{6}+25 q^{5}+16 q^{4}+10 q^{3}+6 q^{2}+3 q+1 .
\end{aligned}
$$

$P_{4}$. The presentation $P_{4}$ is

$$
\left\langle\begin{array}{l|l}
\mathbf{s}, \mathbf{t}, \mathbf{u} & \begin{array}{l}
\mathbf{s t s t}=\mathbf{t s t s}, \text { tutut }=\mathbf{u t u t u}, \text { susus }=\mathbf{u s u s u}, \\
(\mathbf{t u s})^{2} \mathbf{t}=\mathbf{s}(\mathbf{t u s})^{2}, \mathbf{u s}(\mathbf{t u s})^{2}=(\mathbf{s t u})^{2} \mathbf{s u}
\end{array}
\end{array}\right\rangle .
$$

We get $P_{4}$ from $P_{1}$ by taking $\left\{\mathbf{t}, \mathbf{u}, \mathbf{u}^{-1} \mathbf{t}^{-1} \mathbf{s t u}\right\}$ as generators. Its Poincaré polynomial is

$$
\begin{aligned}
& q^{19}+5 q^{18}+16 q^{17}+54 q^{16}+127 q^{15}+211 q^{14}+257 q^{13}+277 q^{12}+288 q^{11}+266 q^{10} \\
& +217 q^{9}+164 q^{8}+117 q^{7}+73 q^{6}+42 q^{5}+23 q^{4}+12 q^{3}+6 q^{2}+3 q+1 .
\end{aligned}
$$


$P_{5}$. Finally, $P_{5}$ just presents the opposite group to $P_{2}$ (the first three relations are the same and each side of the fourth is reversed). It is obtained from $P_{2}$ by exchanging the generators $\mathbf{u}$ and $\mathbf{t}$.

\subsection{Presentations for $B\left(G_{29}\right)$}

For $B\left(G_{29}\right)$, in [5] we considered two presentations on generators $\{\mathbf{s}, \mathbf{t}, \mathbf{u}, \mathbf{v}\}$. These presentations actually correspond to two different presentations of the parabolic subgroup generated by $\{\mathbf{t}, \mathbf{u}, \mathbf{v}\}$ which is of type $B(G(4,4,3))$, so we describe the situation for this last group.

For $B(G(4,4,3))$ a Hurwitz orbit of $\delta$ has 32 elements; two presentations occur along the orbit.

$P_{1}$. The presentation $P_{1}$ is

$$
\left\langle\mathbf{t}, \mathbf{u}, \mathbf{v} \mid \mathbf{t v t}=\mathbf{v t v}, \mathbf{u v u}=\mathbf{v u v}, \mathbf{t u t u}=\mathbf{u t u t},(\mathbf{v u t})^{2}=(\mathbf{u t v})^{2}\right\rangle .
$$

It appears sixteen times in a Hurwitz orbit. Its Poincaré polynomial is

$$
3 q^{8}+13 q^{7}+23 q^{6}+22 q^{5}+15 q^{4}+10 q^{3}+6 q^{2}+3 q+1 .
$$

$P_{2}$. The presentation $P_{2}$ is

$$
\left\langle\mathbf{t}, \mathbf{u}, \mathbf{v} \mid \mathbf{t v t}=\mathbf{v t v}, \mathbf{u v u}=\mathbf{v u v}, \mathbf{t u t}=\mathbf{u t u}, \mathbf{v t}(\mathbf{u v t})^{2}=(\mathbf{u v t})^{2} \mathbf{u v}\right\rangle .
$$

We get $P_{2}$ from $P_{1}$ by taking $\left\{\mathbf{v}^{-1} \mathbf{t v}, \mathbf{u}, \mathbf{v}\right\}$ as generators. It appears eight times in a Hurwitz orbit. Its Poincaré polynomial is

$$
11 q^{8}+21 q^{7}+18 q^{6}+15 q^{5}+12 q^{4}+9 q^{3}+6 q^{2}+3 q+1
$$

A presentation of $B\left(G_{29}\right)$ can be obtained in each case by adding one generator $\mathbf{s}$ and the extra relations $\mathbf{s t s}=\mathbf{t s t}, \mathbf{s u}=\mathbf{u s}, \mathbf{s v}=\mathbf{v s}$.

\subsection{Presentations for $B\left(G_{33}\right)$ and $B\left(G_{34}\right)$}

For $B\left(G_{33}\right)$, in [5] we considered two presentations on generators $\{\mathbf{s}, \mathbf{t}, \mathbf{u}, \mathbf{v}, \mathbf{w}\}$ and for $B\left(G_{34}\right)$ two presentations on generators $\{\mathbf{s}, \mathbf{t}, \mathbf{u}, \mathbf{v}, \mathbf{w}, \mathbf{x}\}$. These presentations differ only on the parabolic subgroup generated by $\{\mathbf{t}, \mathbf{u}, \mathbf{v}, \mathbf{w}\}$ which is of type $B(G(3,3,4))$, so we describe the situation for this last group.

For $B(G(3,3,4))$ a Hurwitz orbit of $\delta$ has 243 elements. Five different presentations occur along the orbit.

$P_{1}$. The presentation $P_{1}$ is

$$
\left\langle\begin{array}{l|l}
\mathbf{t}, \mathbf{u}, \mathbf{v}, \mathbf{w} & \begin{array}{l}
\mathbf{u t u}=\mathbf{t u t}, \mathbf{v t v}=\mathbf{t v t}, \mathbf{v u v}=\mathbf{u v u}, \mathbf{v w v}=\mathbf{w v w}, \\
\mathbf{t w}=\mathbf{w t}, \mathbf{u w}=\mathbf{w u},(\mathbf{v t u})^{2}=(\mathbf{u v t})^{2}
\end{array}
\end{array} .\right.
$$

It appears 108 times in a Hurwitz orbit. Its Poincaré polynomial is

$$
8 q^{12}+40 q^{11}+82 q^{10}+108 q^{9}+109 q^{8}+95 q^{7}+79 q^{6}+57 q^{5}+35 q^{4}+20 q^{3}+10 q^{2}+4 q+1 .
$$

$P_{2}$. The presentation $P_{2}$ is

$$
\left\langle\begin{array}{l|l}
\mathbf{t}, \mathbf{u}, \mathbf{v}, \mathbf{w} & \begin{array}{l}
\mathbf{w t w}=\mathbf{t w t}, \mathbf{u t u}=\mathbf{t u t}, \mathbf{u v u}=\mathbf{v u v}, \mathbf{w v w}=\mathbf{v w v}, \\
\mathbf{t v}=\mathbf{v t}, \mathbf{w u}=\mathbf{u w}, \mathbf{v}(\mathbf{w t u v})^{2}=(\mathbf{w t u v})^{2} \mathbf{w}
\end{array}
\end{array}\right\rangle
$$

It appears nine times in a Hurwitz orbit. Its Poincaré polynomial is

$$
q^{12}+20 q^{11}+74 q^{10}+128 q^{9}+130 q^{8}+100 q^{7}+74 q^{6}+52 q^{5}+34 q^{4}+20 q^{3}+10 q^{2}+4 q+1 .
$$

We get $P_{2}$ from $P_{1}$ by taking $\left\{\mathbf{t}, \mathbf{v}, \mathbf{w}, \mathbf{w}^{-1} \mathbf{v}^{-1} \mathbf{u v w}\right\}$ as generators. 
$P_{3}$. The presentation $P_{3}$ is

$$
\left\langle\begin{array}{l|l}
\mathbf{t}, \mathbf{u}, \mathbf{v}, \mathbf{w} & \begin{array}{l}
\mathrm{tw}=\mathrm{wt}, \mathbf{u w u}=\mathrm{wuw}, \mathbf{u v u}=\mathbf{v u v}, \mathbf{v w v}=\mathbf{w v w}, \mathbf{t u t}=\mathbf{u t u}, \\
\mathbf{t v t}=\mathbf{v t v}, \mathbf{u v w u}=\mathbf{w u v w},(\mathbf{t u v})^{2}=(\mathbf{v t u})^{2}
\end{array}
\end{array}\right\} .
$$

It appears 72 times in a Hurwitz orbit. Its Poincaré polynomial is

$$
34 q^{10}+88 q^{9}+122 q^{8}+132 q^{7}+111 q^{6}+75 q^{5}+45 q^{4}+25 q^{3}+11 q^{2}+4 q+1 .
$$

We get $P_{3}$ from $P_{1}$ by taking $\left\{\mathbf{t}, \mathbf{v}, \mathbf{v}^{-1} \mathbf{u v}, \mathbf{w}\right\}$ as generators.

$P_{4}$. The presentation $P_{4}$ is

$$
\left\langle\begin{array}{l|l}
\mathbf{t}, \mathbf{u}, \mathbf{v}, \mathbf{w} & \begin{array}{l}
\mathbf{t v t}=\mathbf{v t v}, \mathbf{u w u}=\mathbf{w u w}, \mathbf{t w t}=\mathbf{w t w}, \mathbf{t w v t}=\mathbf{v t w v}, \mathbf{w v u w}=\mathbf{u w v u}, \\
\mathbf{t u t}=\mathbf{u t u}, \mathbf{w v w}=\mathbf{v w v}, \mathbf{u v u}=\mathbf{v u v},(\mathbf{t u w})^{2}=(\mathbf{w t u})^{2},(\mathbf{t u v})^{2}=(\mathbf{u v t})^{2}
\end{array}
\end{array}\right\rangle .
$$

It appears 36 times in a Hurwitz orbit. Its Poincaré polynomial is

$$
6 q^{10}+40 q^{9}+98 q^{8}+148 q^{7}+149 q^{6}+102 q^{5}+58 q^{4}+30 q^{3}+12 q^{2}+4 q+1 .
$$

We get $P_{4}$ from $P_{1}$ by taking $\left\{\mathbf{t}, \mathbf{u}, \mathbf{v}, \mathbf{v w v ^ { - 1 }}\right\}$ as generators.

$P_{5}$. The presentation $P_{5}$ is

$$
\left\langle\begin{array}{l|l}
\mathbf{t}, \mathbf{u}, \mathbf{v}, \mathbf{w} & \begin{array}{l}
\mathbf{w u}=\mathbf{u w}, \mathbf{t v t}=\mathbf{v t v}, \mathbf{v u v}=\mathbf{u v u}, \mathbf{t u t}=\mathbf{u t u}, \mathbf{t w t}=\mathbf{w t w}, \\
\mathbf{w v w}=\mathbf{v w v}, \mathbf{t w v u t w}=\mathbf{u t w v u t},(\mathbf{v u t})^{2}=(\mathbf{u t v})^{2},(\mathbf{w v t})^{2}=(\mathbf{v t w})^{2}
\end{array}
\end{array} .\right.
$$

It appears eighteen times in a Hurwitz orbit. Its Poincaré polynomial is

$$
q^{10}+28 q^{9}+97 q^{8}+163 q^{7}+162 q^{6}+104 q^{5}+52 q^{4}+25 q^{3}+11 q^{2}+4 q+1 .
$$

We get $P_{5}$ from $P_{1}$ by taking $\left\{\mathbf{t}, \mathbf{v}, \mathbf{u}, \mathbf{u v w} \mathbf{v}^{-1} \mathbf{u}^{-1}\right\}$ as generators.

In each case we obtain a presentation of $B\left(G_{33}\right)$ by adding one generator $\mathbf{s}$ and the relations $\mathbf{s t s}=\mathbf{t s t}, \mathbf{s u}=\mathbf{u s}, \mathbf{s v}=\mathbf{v s}, \mathbf{s w}=\mathbf{w s}$. We then obtain a presentation of $B\left(G_{34}\right)$ by adding one generator $\mathbf{x}$ and the relations $\mathbf{w} \mathbf{x w}=\mathbf{x w} \mathbf{x}, \mathbf{x s}=\mathbf{s x}, \mathbf{x t}=\mathbf{t x}, \mathbf{x u}=\mathbf{u x}, \mathbf{x v}=\mathbf{v x}$, except for the representation corresponding to $P_{2}$ where the relations we should add are $\mathbf{w x}=\mathbf{x w}, \mathbf{s x s}=\mathbf{x s x}$, $\mathbf{x t}=\mathbf{t} \mathbf{x}, \mathbf{x u}=\mathbf{u x}, \mathbf{x v}=\mathbf{v x}$ (this presentation can be obtained from the one corresponding to $P_{1}$ by taking $\mathbf{s t u t}^{-1} \mathbf{s}^{-1}, \mathbf{s}, \mathbf{t}, \mathbf{v}, \mathbf{w}, \mathbf{x}$ as generators).

\section{Representations from $W$-graphs}

The notion of a $W$-graph for a representation of a Weyl group originates from Kazhdan-Lusztig theory. Here, we propose a generalization of this concept to the case of complex reflection groups. We then deal with computational issues connected with this.

\section{1. $W$-graphs}

Let $W \leqslant \mathrm{GL}(V)$ be a complex reflection group on $V$. We assume that $W$ is well-generated; that is, $W$ can be generated by $n:=\operatorname{dim}(V)$ reflections $s_{1}, \ldots, s_{n}$. We set $I=\{1,2, \ldots, n\}$ and we let $d_{j}$ denote the order of the reflection $s_{j}, j \in I$, and $d:=\max \left\{d_{j}\right\}$.

The following generalizes the concept of a $W$-graph for a representation of a finite Coxeter group; see [12]. Let $R: W \rightarrow \mathrm{GL}_{r}(\mathbb{C})$ be an irreducible representation of $W$. A pre- $W$-graph $\Gamma$ for $R$ is a sequence $\left(\gamma_{1}, \ldots, \gamma_{r}\right)$ of $r$ maps $\gamma_{k}: I \rightarrow\{0, \ldots, d-1\}$ satisfying $\gamma_{k}(j) \leqslant d_{j}-1$ for all $1 \leqslant k \leqslant r, j \in I$. The maps $\gamma_{k}$ are also called the nodes of $\Gamma$.

We now define the concept of an admissible pre- $W$-graph. If $W$ is cyclic, then $n=1$ and $I=$ $\{1\}$. Any irreducible representation is one-dimensional, so $r=1$, and the generating reflection $s_{1}$ acts by $R\left(s_{1}\right)=\exp \left(2 \pi i m / d_{1}\right)$ in $R$ for some $1 \leqslant m \leqslant d_{1}$. Then only the map $\gamma_{1}$ with $\gamma_{1}(1)=m$ is admissible. Now assume inductively that an admissible pre- $W$-graph has been chosen for each 
irreducible representation of each proper parabolic subgroup $W_{J}=\left\langle s_{j} \mid j \in J\right\rangle$, where $J \subset I$. The pre- $W$-graph $\Gamma$ is then called admissible (with respect to the chosen admissible pre- $W$ graphs of the parabolics), if for each parabolic subgroup $W_{J}<W$ the restriction of $\Gamma$ to $W_{J}$ is the union of the pre- $W$-graphs of the restriction of $R$ to $W_{J}$. (Here, restriction to a parabolic subgroup $W_{J}, J \subset I$, is obtained by restricting all $\gamma_{j}$ to $J$.)

Let $\mathcal{H}(W, \mathbf{u})$ denote the generic cyclotomic Hecke algebra associated to $W$, where $\mathbf{u}=$ $\left(u_{j, m} \mid 1 \leqslant j \leqslant n, 0 \leqslant m \leqslant d_{j}-1\right)$ with $u_{j, 0}, \ldots, u_{j, d_{j}-1}$ corresponding to $s_{j}$ as above. For each $j$ we choose a total ordering on the variables $u_{j, 0}, \ldots, u_{j, d_{j}-1}$; for example $u_{j, 0}>u_{j, 1}>$ $\ldots>u_{j, d_{j}-1}$. We write $T_{j}$ for the image in $\mathcal{H}(W, \mathbf{u})$ of a braid reflection mapping to $s_{j}$, $1 \leqslant j \leqslant n$. Given an admissible pre- $W$-graph $\Gamma$ for a representation $R$ of $W$, we associate a pre-representation of $\mathcal{H}(W, \mathbf{u})$ as follows. For each $j \in I$, let $T_{j}$ be an $(r \times r)$-matrix with diagonal entries $T_{j}[k, k]=u_{j, \gamma_{k}(j)}$. The off-diagonal entry $T_{j}[k, l]$ is zero unless $T_{j}[k, k]<T_{j}[l, l]$ in the chosen ordering on $\mathbf{u}$. The remaining entries of the $T_{j}$ are independent indeterminates, except that $T_{j}[k, l]=T_{m}[k, l]$ if $s_{j}, s_{m}$ are conjugate in $W$ and both $T_{j}[k, k]=T_{m}[k, k]$ and $T_{j}[l, l]=T_{m}[l, l]$, for $k \neq l$.

Any specialization of these matrices which define a representation of $\mathcal{H}(W, \mathbf{u})$ which is a deformation of a conjugate of the given representation $R$ of $W$ is called a $W$-graph for $R$. Note that, since $W$ is admissible, the characteristic polynomials of the $T_{j}$ are by construction already as they should be if the $T_{j}$ did define a representation of $\mathcal{H}(W)$ specializing to $R$.

If all reflections of $W$ are conjugate and of order two, such a representation can be encoded in an actual labelled and directed graph as follows: the nodes of the graph are in bijection with the set $\{1, \ldots, r\}$, labelled by $\gamma_{k}(1)$ (note that here $\gamma_{k}$ is already uniquely determined by $\left.\gamma_{k}(1)\right)$. There is a directed edge from $j$ to $k$, labelled by $T_{m}[j, k]$, if $T_{m}[j, k] \neq 0$ for some $m$ with $\gamma_{j}(m)=1$ and $\gamma_{k}(m)>1$. Note that the value of $T_{m}[j, k]$ does not depend on the choice of $m$, by our convention on pre-representations. Clearly the representation can be recovered from this graph. Note also that there are just two possible total orderings of the two variables $u_{0}, u_{1}$ in this case, and changing the ordering amounts to transposing the representing matrices.

For instance, here is the graph for the representation of $\mathcal{H}(W, \mathbf{u})$ specializing to the reflection representation, where $W=G_{24}, \mathbf{u}=\left\{u_{1,0}, u_{1,1}\right\}=\{x, y\}$.

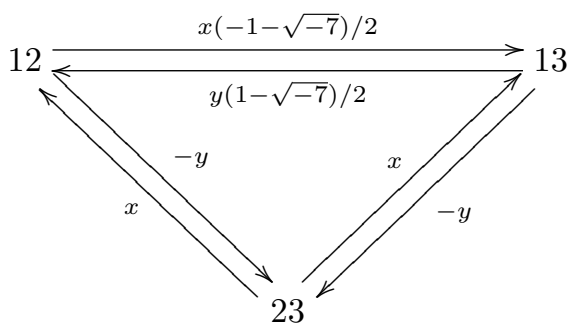

We consider the matrices for the three generators for presentation $P_{1}$ in $\S 6.1$. We have $d=2$, $r=3$.

REMARK 7.2. Assume for a moment that $W$ is a real reflection group, that is, a finite Coxeter group. In this case Gyoja has shown [12] that any irreducible representation has a model which comes from a pre-representation of a $W$-graph of $W$.

In general, for arbitrary complex reflection groups, admissible pre- $W$-graphs need not always exist. But in cases where pre- $W$-graphs do exist, examples show that:

(a) often, there exist corresponding representations;

(b) these representations tend to be very sparse;

(c) often the entries can be chosen to be Laurent-polynomials in the parameters;

(d) all $n$ matrices are equally sparse. 


\subsection{Existence of pre- $W$-graphs and $W$-graphs}

We say that a representation of $W$ has an admissible pre- $W$-graph if there exists one for some presentation of the braid group and for some ordering of the variables. It is pretty straightforward to write a program which enumerates all admissible pre- $W$-graphs for $W$, given those of the proper parabolic subgroups. In order for this inductive process to work, we have to also consider some rank two groups.

It is much more difficult in general to find, given a pre-representation for a pre- $W$-graph of $W$, specializations of the entries so that it actually defines a representation of $\mathcal{H}(W)$ (which specializes to the representation $R$ of $W$ we started with).

Let us give some indications on how the $W$-graphs presented below were constructed. For small representations (of dimension at most four) this is straightforward, and is done by solving the non-linear system of equations obtained by requiring that the given pre-representation satisfies the relations of $\mathcal{H}:=\mathcal{H}(W, \mathbf{u})$. For larger dimensions, this system becomes too large: if the representation $R$ has dimension $r$ and $\mathcal{H}$ has $n$ generators, then the matrices of the generators for the pre-representation involve at least roughly $n r^{2} / 4$ unknowns. A braid relation of length $m$ in the generators produces algebraic equations of degree $m$ between these unknowns. Furthermore, the coefficients in the equations involve all the parameters $\mathbf{u}$ of the Hecke algebra. A simple minded application of the Buchberger algorithm to such a system of equations is bound to fail.

Therefore, we had to use several tricks. A look at the final representations shows that they are very sparse, containing many more zero-entries than required by the definition of prerepresentation. Knowing the positions of these zeros in advance would allow us to solve the system of equations easily. Thus, in a first step, we tried to conjugate the given representation of $W$ into the form of a $W$-graph, with as many zeros as possible. Note that the conditions on the entries of such a conjugating matrix are linear, hence this system is easy to solve. In general, there will not be a unique solution, but we chose a solution with as many zeros as possible. Then we looked for a $W$-graph representation of $\mathcal{H}$ with zero entries in the same positions.

Alternatively, we started from a representation of $\mathcal{H}$ obtained by the methods of $\S 5$, for example, and tried to conjugate this to a $W$-graph representation.

For dimensions larger than ten, say, even the determination of such a conjugating matrix becomes too difficult. Here, one successful approach used information from maximal parabolic subgroups. Let $W_{J}$ be a maximal parabolic subgroup of $W$, and assume that the restriction of $R$ to $W_{J}$ splits as

$$
\left.R\right|_{W_{J}}=R_{1} \oplus \ldots \oplus R_{t}
$$

into a sum of irreducible representations $R_{i}$ of $W_{J}$. By induction, we may assume that $W$-graphs of the $R_{i}$ for $\mathcal{H}\left(W_{J}\right)$ are already known. In order to use this information we made the additional assumption that the block diagonal part of the $T_{j}$, with $j \in J$, agrees with the $W$-graphs of the $R_{i}$. (This does not follow from our axioms on pre- $W$-graphs, and it is in fact not always satisfied.) This 'Ansatz' again reduces the number of unknowns considerably. Clearly, the larger the dimensions of the $R_{i}$ are, that is, the fewer constituents occur, the more information we obtain.

7.2.1. Some rank-two groups. We describe the situation in some detail for the case of the smallest well-generated exceptional group $G_{4}$. There is for each representation exactly one $W$-graph, as given in Table 2. The labelling of characters is as in [15], for example.

Each two-dimensional representation admits one further pre- $W$-graph, for instance $\phi_{2,5}$ admits $(.1 .2, .2 .1)$; however the only $W$-graph corresponding to it is a non-irreducible representation (which has the same restriction to parabolic subgroups). For the three-dimensional 
representation, there are five more pre- $W$-graphs:

$$
(. .12, .12 ., 12 . .), \quad(. .12,1.2 ., 2.1 .), \quad(.1 .2, .2 .1,12 . .), \quad(.1 .2,1.2 ., 2 . .1), \quad(.2 .1,1 . .2,2.1 .) \text {. }
$$

The first three give rise to non-irreducible representations, and the last two do not give rise to any representation.

Similarly, for the group $G_{3,1,2}$, each representation admits one pre- $W$-graph which is a $W$ graph, except for the two-dimensional representations which also admit another pre- $W$-graph giving rise to a non-irreducible representation. The same situation holds for the Coxeter groups $A_{2}, B_{2}$ and $I_{2}(5)$.

7.2.2. Pre- $W$-graphs for $G_{25}$. The inductive approach now gives the following proposition.

Proposition 7.3. For $G_{25}$, each irreducible representation admits a single pre- $W$-graph whose restriction to each parabolic subgroup $G_{4}$ is an actual $W$-graph. For each pre- $W$-graph, there exists a corresponding $W$-graph.

Table 3 contains these $W$-graphs for one representation in each orbit under Galois automorphisms on the parameters.

\subsubsection{Pre-W-graphs for $G_{26}$.}

Proposition 7.4. For $G_{26}$, all but two six-dimensional irreducible representations admit at least one pre- $W$-graph whose restrictions to both parabolic subgroups of type $G_{4}$ and $G_{3,1,2}$ are actually $W$-graphs. For each pre- $W$-graph, there exists a corresponding $W$-graph.

Table 4 contains the unique pre- $W$-graph for the given representatives of the Galois orbits.

TABLE $2 . W$-graphs for $G_{4}$.

\begin{tabular}{cccc}
\hline Character & $W$-graph & Character & $W$-graph \\
\hline$\phi_{1,0}$ & $(12 .)$. & $\phi_{2,1}$ & $(.12 ., 12 .)$. \\
$\phi_{1,4}$ & $(.12)$. & $\phi_{2,3}$ & $(.12,12 .)$. \\
$\phi_{1,8}$ & $(. .12)$ & $\phi_{2,5}$ & $(. .12, .12)$. \\
$\phi_{3,2}$ & $(.12 ., 1 . .2,2 . .1)$ & & \\
\hline
\end{tabular}

TABLE $3 . W$-graphs for $G_{25}$.

\begin{tabular}{cc}
\hline Character & $W$-graph \\
\hline$\phi_{1,0}$ & $(123 .)$. \\
$\phi_{2,3}$ & $(13.2 ., 2.13)$. \\
$\phi_{3,6}$ & $(.123 ., 13 . .2,2 . .13)$ \\
$\phi_{3,1}$ & $(12.3 ., 13.2 ., 23.1)$. \\
$\phi_{6,2}$ & $(1.23 ., 12 . .3,13 . .2,2.13 ., 23 . .1,3.12)$. \\
$\phi_{6,4}^{\prime \prime}$ & $(.123 ., 1.23 ., 13 . .2,2.1 .3,2.3 .1,3.12)$. \\
$\phi_{8,3}$ & $(1.23 ., 12 . .3,13 . .2,13 . .2,2.1 .3,2.3 .1,23 . .1,3.12)$. \\
$\phi_{9,5}$ & $(.123 ., 1.2 .3,1.3 .2,13 . .2,2 . .13,2.1 .3,2.3 .1,3.1 .2,3.2 .1)$ \\
\hline
\end{tabular}

TABLE 4 . $W$-graphs for $G_{26}$.

\begin{tabular}{cc}
\hline Character & $W$-graph \\
\hline$\phi_{1,0}$ & $(123 .)$. \\
$\phi_{2,3}$ & $(12.3 ., 13.2)$. \\
$\phi_{3,1}$ & $(12.3 ., 13.2 ., 23.1)$. \\
$\phi_{3,6}$ & $(1.23 ., 12 . .3,13 . .2)$ \\
$\phi_{6,2}$ & $(1.23 ., 12 . .3,13.22,13.2 ., 2.13 ., 23.1)$. \\
$\phi_{8,3}$ & $(1.2 .3,1.23 . ., 1.3 .2,12 . .3,13 . .2,13.2 ., 2.1 .3 .13 .13 .1 .2 .13 .1 .2)$ \\
$\phi_{9,5}$ &
\end{tabular}


7.2.4. Pre- $W$-graphs for $G_{24}$. For $G_{24}$, the situation depends on the presentation of the braid group $B\left(G_{24}\right)$ considered; see $\S 6.1$.

Proposition 7.5. For $G_{24}$, for each of the presentations $P_{1}$ to $P_{3}$, each representation admits at most one pre- $W$-graph whose restrictions to parabolic subgroups of type $A_{2}$ and $B_{2}$ are $W$-graphs. But for $P_{2}$ and $P_{3}$ (the same) eight of the twelve representations admit such a graph, while for $P_{1}$ two more representations admit such pre- $W$-graphs. For each pre- $W$-graph for $P_{1}$, there exists a corresponding $W$-graph.

Table 5 contains the pre- $W$-graphs for the given representatives of the Galois orbits and presentation $P_{1}$.

The representations $\phi_{8,4}$ and $\phi_{8,5}$ do not admit any pre- $W$-graph.

A $W$-graph for $\phi_{3,1}$ has been given in (7.1). With the same conventions, here is a $W$-graph for $\phi_{6,2}$ :

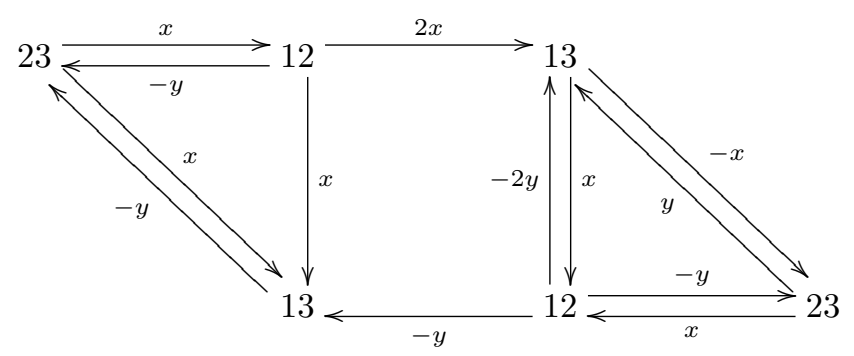

and here is a $W$-graph for $\phi_{7,6}$.

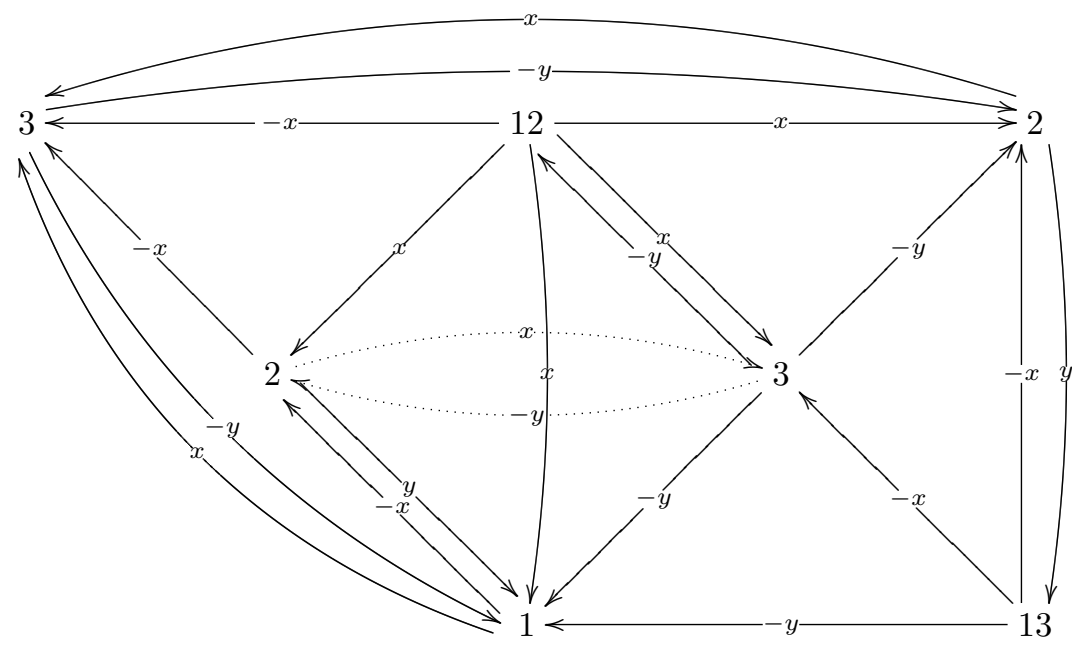

Together with the matrices given above for $\phi_{8,5}$, this completes the description of the representations of the Hecke algebra of $G_{24}$.

TABLE 5. $W$-graphs for $G_{24}$, presentation $P_{1}$.

\begin{tabular}{cc}
\hline Character & $W$-graph \\
\hline$\phi_{1,0}$ & $(132)$ \\
$\phi_{3,1}$ & $(13,12,32)$ \\
$\phi_{6,2}$ & $(13,13,12,12,32,32)$ \\
$\phi_{7,6}$ & $(1,13,12,3,3,2,2)$ \\
\hline
\end{tabular}




\subsubsection{Pre-W-graphs for $G_{27}$.}

Proposition 7.6. For the presentations $P_{1}$ to $P_{5}$ of $G_{27}$, each representation admits at most one pre- $W$-graph whose restrictions to parabolic subgroups of type $A_{2}, B_{2}$ and $I_{2}(5)$ are $W$-graphs. For $P_{1} 26$ out of 34 representations admit such a graph, while for $P_{2}$ (respectively $\left.P_{3}, P_{4}, P_{5}\right)$ just sixteen (respectively twenty, fourteen, sixteen) admit such a graph. Moreover, any representation which admits such a graph for any of $P_{2}-P_{5}$ admits one for $P_{1}$. For each pre- $W$-graph for $P_{1}$, there exists a corresponding $W$-graph.

Table 6 contains the pre- $W$-graphs for $P_{1}$ for representatives of the Galois orbits. The eightand fifteen-dimensional representations do not admit any pre- $W$-graph.

Here is a $W$-graph for $\phi_{3,1}$, where $c=1+\zeta_{3}^{2}(1-\sqrt{5}) / 2$.

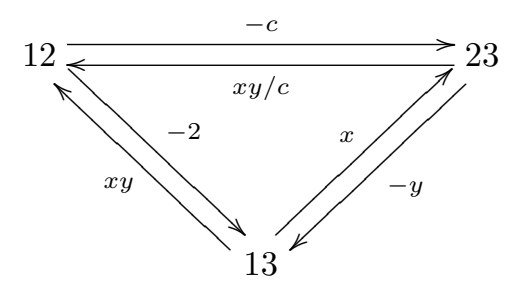

Here is a $W$-graph for $\phi_{5,6}^{\prime}$ :

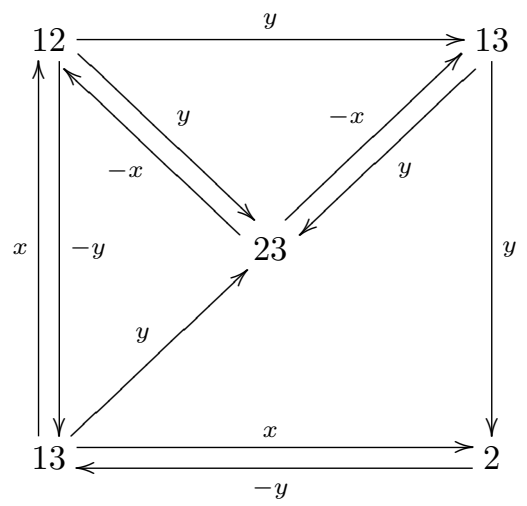

and here is one for $\phi_{6,2}$.

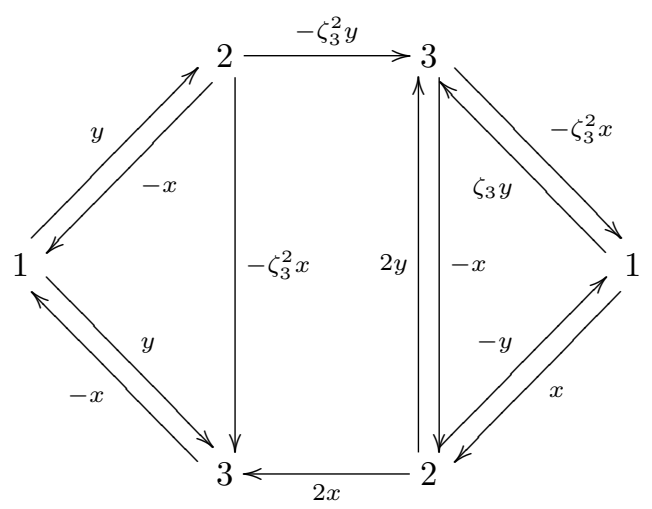

Interchanging the generators $\mathbf{u}, \mathbf{t}$ in the presentation $P_{1}$ obviously defines an antiautomorphism of $B(W)$. It can be checked that composition of this antiautomorphism with transposition interchanges the representations $\phi_{5,6}^{\prime}$ and $\phi_{5,6}^{\prime \prime}$, so we need not give a $W$-graph for the latter. 
We now give a $W$-graph for $\phi_{9,6}$ as the union of the following pieces. The nodes are 1,2 , $3,12,12,13,13,23,23$ (the last three occur twice and we box one of the occurrences to distinguish it from the other). Here we set $u=\sqrt[3]{x}$ and $v=\sqrt[3]{y}$.

$$
\begin{gathered}
3 \stackrel{u^{3}}{\longleftarrow} 12 \stackrel{v^{2}-u v}{\longrightarrow} 13 \stackrel{-u^{3} v}{\longrightarrow} 12 \stackrel{(u-v)\left(u^{2}+v^{2}\right)}{\longrightarrow} 1 \stackrel{-u^{3} v^{3}}{\longrightarrow} 23 \stackrel{1}{\longrightarrow} 2 \stackrel{(u-v)^{2}}{\longrightarrow} 23 \\
13 \stackrel{-v^{2}}{\longleftarrow} 2 \stackrel{v^{2}(u-v)}{\longleftarrow} 12 \stackrel{-u v^{2}}{\longrightarrow} 13 \stackrel{v^{2}(u-v)}{\longrightarrow} 1 \stackrel{1}{\longleftarrow} 23 \stackrel{-1}{\longrightarrow} 3 \stackrel{v(2 u-v)}{\longleftarrow} 23 \stackrel{-u^{2} v^{2}}{\longleftarrow} 12 \\
13 \stackrel{-u^{2} v^{2}}{\longrightarrow} 23 \stackrel{u v}{\longrightarrow} 12 \stackrel{v^{2}}{\longrightarrow} 13 \stackrel{u^{2} v(v-u)}{\longrightarrow} 3 \stackrel{v^{2}(u-v)}{\longrightarrow} 2 \\
2 \stackrel{u^{3} v}{\longleftarrow} 13 \stackrel{u-v}{\stackrel{u}{\longleftarrow}} 23 \stackrel{u v}{\longrightarrow} 13 \stackrel{u^{2} v}{\longrightarrow} 12 \stackrel{u\left(u v-u^{2}-v^{2}\right)}{\longrightarrow} 1 \\
2 \stackrel{u^{2}(v-u)}{\longleftarrow} 13 \stackrel{u^{2}(u-v)}{\longrightarrow} 3 \stackrel{-v^{3}}{\longrightarrow} 12
\end{gathered}
$$

Here is, following the same conventions, a $W$-graph for $\phi_{10,3}$. The nodes are 13, 12, 13 , 12 , 13 , $12,3,2,23,23$.

$$
\begin{aligned}
& 13 \stackrel{y}{\longrightarrow} 3 \stackrel{-1}{\longrightarrow} 12 \stackrel{-y}{\longrightarrow} 13 \stackrel{x y+x^{2}+y^{2}}{\longrightarrow} 12 \stackrel{x}{\longrightarrow} 23 \stackrel{-1}{\longrightarrow} 13 \stackrel{x}{\longrightarrow} \stackrel{12}{\longrightarrow} \stackrel{x-y}{\longrightarrow} 2 \stackrel{-x+2 y}{\longrightarrow} 23
\end{aligned}
$$

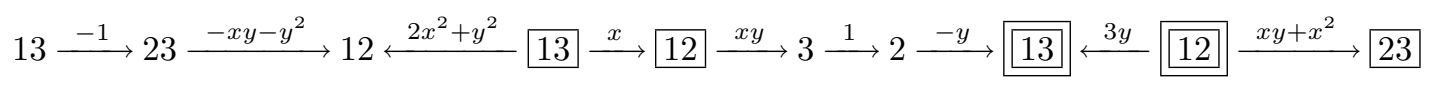

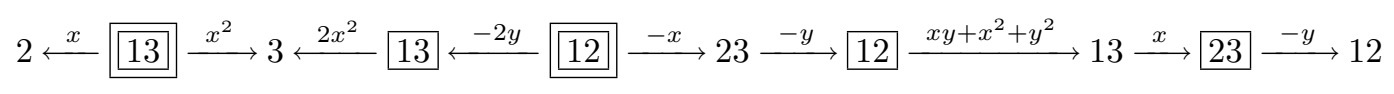

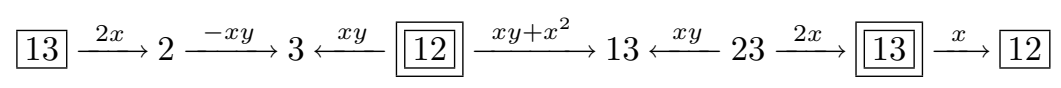

$$
\begin{aligned}
& 12 \stackrel{x+y}{\longrightarrow} 2 \stackrel{-1}{\longleftarrow} 23 \stackrel{x y}{\longleftarrow} 13 \stackrel{-x}{\longleftarrow} 23 \stackrel{y}{\longrightarrow} 12 \\
& 23 \stackrel{y^{2}}{\longrightarrow} 3 \quad 23 \stackrel{2}{\longrightarrow} 13
\end{aligned}
$$

We have found a model for $\phi_{15,5}$ by Hensel lifting with coefficients in Frac $\tilde{A}$, that with meataxe techniques we could reduce to have coefficients in $\tilde{A}$.

We now give some information on higher-dimensional primitive groups.

7.2.6. Pre- $W$-graphs for $G(4,4,3)$ and $G_{29}$. For the presentation of $G(4,4,3)$ corresponding to $P_{1}$ the six-dimensional representation does not admit a pre- $W$-graph while

TABLE 6. $W$-graphs for $G_{27}$.

\begin{tabular}{cc}
\hline Character & $W$-graph \\
\hline$\phi_{1,0}$ & $(132)$ \\
$\phi_{3,1}^{\prime \prime}$ & $(12,13,23)$ \\
$\phi_{5,6}^{\prime \prime}$ & $(12,13,13,2,23)$ \\
$\phi_{5,6}^{\prime}$ & $(12,12,13,23,3.12)$ \\
$\phi_{6,2}$ & $(13,13,12,12,23,23)$ \\
$\phi_{9,6}$ & $(1,12,12,13,13,2,23,23,3)$ \\
$\phi_{10,3}$ & $(12,12,12,13,13,13,2,23,23,3)$ \\
\hline
\end{tabular}


for the presentation corresponding to $P_{2}$ it is the two-dimensional representation which does not admit one.

Proposition 7.7. For the presentation corresponding to $P_{1}$ of $G_{29}$, fifteen representations admit a pre- $W$-graph, while for that corresponding to $P_{2}, 27$ (out of 37) admit one. The two representations $\phi_{5,8}$ and $\phi_{5,16}$ admit a pre- $W$-graph for $P_{1}$ and not for $P_{2}$.

All together, all representations of $W$ admit a pre- $W$ graph except for two of the four of dimension fifteen, and for those of dimension twenty. We have found actual $W$-graphs for all the pre- $W$-graphs except the last three of Table 7.

Table 7 contains pre- $W$-graphs for representatives of Galois orbits of representations of the Hecke algebra. The first seven graphs in the table correspond to $P_{1}$ and the rest to $P_{2}$. To condense the table, repeated nodes are represented once, the multiplicity being given by an exponent.

7.2.7. Pre- $W$-graphs for $G_{32}$. For $G_{32}, 57$ of the 102 irreducible representations admit a pre- $W$-graph. If one includes the Galois-conjugates of these representations, one gets all representations but twelve: the missing ones are three of the 60-dimensional ones, the 64-dimensional and the 81-dimensional ones.

7.2.8. Pre- $W$-graphs for $G_{33}$. For the presentation $P_{1}$ of $\S 6.4$ we find that fourteen representations admit a pre- $W$-graph, and for presentation $P_{2}$ we find that fourteen more admit one, for a total of 28 out of 40 . The set of representations which admit a pre- $W$-graph is stable under Galois action, so we do not get new ones. For the other presentations $P_{3}$ to $P_{5}$ the representations which admit a pre- $W$-graph are a subset of the fourteen which have one for $P_{1}$.

7.2.9. Pre- $W$-graphs for $G_{34}$. For the presentation corresponding to $P_{1}$ of $\S 6.4$ we find that eighteen representations admit a pre- $W$-graph, and for the presentation corresponding to $P_{2}$ we find that thirteen more admit one, for a total of 31 out of 169 . The set of representations which admit a pre- $W$-graph is stable under Galois action, so this does not give new ones. For the presentations corresponding to $P_{3}, P_{4}, P_{5}$ the representations which admit a pre- $W$-graph are a subset of the eighteen which have one for $P_{1}$.

TABle 7. Pre- $W$-graphs for $G_{29}$.

\begin{tabular}{cc}
\hline Character & $W$-graph \\
\hline$\phi_{1,0}$ & $(1234)$ \\
$\phi_{4,4}$ & $(123,124,134,234)$ \\
$\phi_{4,1}$ & $(123,124,134,234)$ \\
$\phi_{5,8}$ & $(123,134,14,23,24)$ \\
$\phi_{6,12}$ & $(12,13,14,23,24,34)$ \\
$\phi_{6,10}^{\prime \prime \prime}$ & $\left(123^{2}, 124^{2}, 134^{3}, 23,234,24\right)$ \\
$\phi_{10,2}$ & $(13,134,142,32,4,2)$ \\
\hline$\phi_{6,10}^{\prime}$ & $(13,134,132,14,142,12,34,342,32,42)$ \\
$\phi_{10,6}$ & $\left(13,134^{3}, 132^{2}, 14,142^{2}, 34,32^{2}, 42^{2}, 2\right)$ \\
$\phi_{15,4}^{\prime}$ & $\left(13^{3}, 134^{2}, 132,132^{2}, 14,144^{3}, 142,12^{2}, 3,34,34^{2}, 32^{3}, 4,42^{3}, 2^{2}\right)$ \\
$\phi_{16,3}$ & $\left(13^{3}, 134^{2}, 132,14^{3}, 142,12^{2}, 3,34^{2}, 32^{3}, 4,42^{3}, 2^{2}\right)$ \\
$\phi_{24,6}$ & $\left(13^{4}, 134^{2}, 132,14^{4}, 142,12^{3}, 3,34^{3}, 32^{4}, 4,42^{4}, 2^{2}\right)$ \\
$\phi_{24,7}$ & \\
$\phi_{30,8}$ &
\end{tabular}




\section{Checking the conjectures of $\S 2$}

We now use the representations obtained above for two- and three-dimensional exceptional groups in order to verify some of the conjectures on the structure of cyclotomic Hecke algebras stated in $\S 2$ for some of the primitive complex reflection groups.

\subsection{Computational difficulties}

The main problem in carrying out the computations implied by, for example Proposition 2.10 is to compute the form $t$ on a large set of images of elements of $B(W)$ (a set of cardinality $r|W|^{2}$, where $r$ is the rank of $W$ ).

To compute $t$, we use formula (2.4), where the Schur elements are taken from [15] and $\chi(x)$ is computed using the matrices for the representation of character $\chi$ that we computed in the previous sections.

To minimize the computations, we use a few tricks:

- we compute the orbits of the set of words we consider under the braid relations and rotations (which give a conjugate element in the braid group). It is sufficient to compute the trace on one element of each orbit;

- when all generators of $W$ are of order two, if in one of the orbits we have a word of length $k$ where there is a repetition ....ss . . . using the quadratic defining relation of the Hecke algebra $\left(T_{\mathbf{s}}-u_{s, 0}\right)\left(T_{\mathbf{s}}-u_{s, 1}\right)=0$ we can reduce the computation to that for one word of length $k-1$ and one word of length $k-2$.

For instance, for $G_{24}$ to compute the matrix $\left\{t\left(T_{\mathbf{w w}^{\prime}}\right)\right\}$ we have to compute the trace on $336^{2}=112896$ elements; they fall into 14334 orbits under rotations and braid relations, and after taking into account quadratic relations we still have to handle 327 elements. For computing the matrix products corresponding to these words, we look for the occurrence of common subwords so as to never compute the same product twice, which means that for each representation we have about 600 matrix products to effect. We also take into account the Galois action on representations so we need to compute the character value only for one representation in each Galois orbit; for $G_{24}$ there are five such orbits.

Even with these simplifications, the matrix products for algebras which have many parameters get very costly, as well as the final step of evaluating the right-hand side of (2.4), since the gcd of the Schur elements is a large polynomial. In quite a few cases we could only make a heuristic check, by computing in the algebra where the parameters are specialized to prime powers (to primes taken to the eth power, so the algebra splits over $\mathbb{Q}$ ). The heuristic check for belonging to $A$ becomes to belong to $\mathbb{Z}$ localized at the chosen primes, and to be a unit in $A$ becomes being an integer with only prime factors the chosen primes.

\subsection{Finding a section such that $t\left(T_{\mathbf{w}}\right)=0$}

In order to find a section $\mathbf{W} \subset B(W)$ such that $t\left(T_{\mathbf{w}}\right)=0$ for any $\mathbf{w} \neq 1$, our first idea was, mimicking the case of finite Coxeter groups, to lift elements of $W$ by lifting minimal length expressions for them as positive words in the generators $s_{1}, \ldots, s_{n}$. However, though this almost works, it does not always work; we manage with a slight variation on this, as we shall explain.

Obtaining all minimal length expressions for elements of $W$ is quite easy using standard methods for enumerating elements of a group, and is feasible for all exceptional complex reflection groups but $G_{34}$.

Tables 8 and 9 collect in the column ' $t\left(T_{\mathbf{w}}\right) \neq 0$ ' the results we obtained by computing the trace on minimal length elements. The number in the column is the number of elements $w \in W$ such that some minimal word $\mathbf{w}$ for $w$ has $t\left(T_{\mathbf{w}}\right) \neq 0$. If this number is not 0 , the second number separated by a / is the number of elements $w \in W$ such that no minimal word $\mathbf{w}$ for $w$ has $t\left(T_{\mathbf{w}}\right)=0$. 
When some minimal word for any $w \in W$ has zero trace, we build a section by choosing arbitrarily such a word for each element. We now describe how to build a section in the other cases.

For $G_{11}$, with the notation of $\S 4$, all minimal length expressions of the two elements $s_{3}^{2}\left(s_{2} s_{1} s_{3}\right)^{2},\left(s_{2} s_{1} s_{3}\right)^{2} s_{3} s_{2}$ have non-zero trace; but the longer lifts $\mathbf{s}_{1} \mathbf{s}_{2} \mathbf{s}_{1} \mathbf{s}_{3} \mathbf{s}_{2} \mathbf{s}_{1} \mathbf{s}_{3}^{2} \mathbf{s}_{1} \mathbf{s}_{3}$, $\mathbf{s}_{1} \mathbf{S}_{3} \mathbf{s}_{2} \mathbf{s}_{1} \mathbf{s}_{3}^{2}\left(\mathbf{s}_{2} \mathbf{s}_{1}\right)^{2}$ have a zero trace. By making these picks, we can find a section which satisfies $t\left(T_{\mathbf{w}}\right)=t\left(T_{\mathbf{w}^{-1} \boldsymbol{\pi}}\right)=0$.

For $G_{15}$, all minimal length expressions of the two elements $s_{2}\left(s_{1} s_{3}\right)^{2} s_{2}, s_{2} s_{1}\left(s_{3} s_{2}\right)^{2}$ have $t\left(T_{\mathbf{w}}\right) \neq 0$; but the longer lifts $\left(\mathbf{s}_{2} \mathbf{s}_{2} \mathbf{s}_{3}\right)^{2} \mathbf{s}_{2}, \mathbf{s}_{2}\left(\mathbf{s}_{1} \mathbf{s}_{3}\right)^{2} \mathbf{s}_{1} \mathbf{s}_{2}^{2}$ have a zero trace. All minimal expressions of $\mathbf{s}_{1} \mathbf{s}_{2}\left(\mathbf{s}_{3} \mathbf{s}_{2}\right)^{2}, \mathbf{s}_{1} \mathbf{s}_{2}\left(\mathbf{s}_{2} \mathbf{s}_{3}\right)^{2},\left(\mathbf{s}_{3} \mathbf{s}_{2}\right)^{2} \mathbf{s}_{1} \mathbf{s}_{2}$ have $t\left(T_{\mathbf{w}^{-1} \boldsymbol{\pi}}\right) \neq 0$. But the longer lifts $\mathbf{s}_{1}\left(\mathbf{s}_{3} \mathbf{s}_{2}\right)^{3} \mathbf{s}_{3},\left(\mathbf{s}_{3} \mathbf{s}_{2}\right)^{3} \mathbf{s}_{3} \mathbf{s}_{1}, \mathbf{s}_{3}\left(\mathbf{s}_{2} \mathbf{s}_{1}\right)^{3} \mathbf{s}_{3}$ work. By making these picks, we can find a section which satisfies $t\left(T_{\mathbf{w}}\right)=t\left(T_{\mathbf{w}^{-1} \boldsymbol{\pi}}\right)=0$ and $t\left(T_{\mathbf{w w}^{\prime}}\right) \in A$, but unfortunately $\operatorname{det}\left\{t\left(T_{\mathbf{w} \mathbf{w}^{\prime}}\right)\right\}_{w, w^{\prime}}$ is not invertible in $A$ for this choice.

However, there is another way to build a section which leads to a good section. Since $G_{11}$ and $G_{15}$ have the same hyperplane arrangements, the braid group $B\left(G_{15}\right)$ is a subgroup of index 2 of $B\left(G_{11}\right)$, generated by $\mathbf{s}_{1}, \mathbf{s}_{2}, \mathbf{s}_{3}^{2}$. The elements in the above section for $G_{11}$ where $\mathbf{s}_{3}$ occurs an even number of times form a section for $G_{15}$ which turns out to be good.

For $G_{24}$ and $G_{27}$ we only consider the presentation $P_{1}$ as it is the best behaved. For $G_{27}$, all minimal length expressions of the element $(s u t)^{5}$ have non-zero trace. The center of $B(W)$ is generated by the element $\mathbf{z}=(\mathbf{s t u})^{5}$. The 'bad' element (sut $)^{5}$ is a lift of $z^{-1}$. The lift $\mathbf{z}^{5}$ of $z^{-1}$, which is much longer, satisfies $t\left(T_{\mathbf{z}^{5}}\right)=0$.

TABLE 8. Hecke algebras for two-dimensional primitive groups.

\begin{tabular}{ccccccccc}
\hline$W$ & $|W|$ & $|\mathbf{u}|$ & Algebra & $t\left(T_{\mathbf{w}}\right) \neq 0$ & $t\left(T_{\mathbf{w}}-1 \boldsymbol{\pi}\right) \neq 0$ & Good & Rank [18] & Form [18] \\
\hline$G_{4}$ & 24 & 3 & + & 0 & 0 & + & + & + \\
$G_{5}$ & 72 & 6 & Specialized & 0 & 0 & + & + & + \\
$G_{6}$ & 48 & 5 & Specialized & 0 & 0 & + & + & + \\
$G_{7}$ & 144 & 8 & Specialized & $3 / 0$ & $1 / 0$ & + & + & + \\
$G_{8}$ & 96 & 4 & Specialized & 0 & 0 & + & + & + \\
$G_{9}$ & 192 & 6 & Specialized & 0 & 0 & + & + & + \\
$G_{10}$ & 288 & 7 & Specialized & $2 / 0$ & $2 / 0$ & + & + & + \\
$G_{11}$ & 576 & 9 & Specialized & $22 / 2$ & $12 / 0$ & + & + & $?$ \\
$G_{12}$ & 48 & 2 & + & 0 & 0 & + & + & + \\
$G_{13}$ & 96 & 4 & Specialized & $1 / 0$ & 0 & + & + & + \\
$G_{14}$ & 144 & 5 & Specialized & 0 & 0 & + & + & + \\
$G_{15}$ & 288 & 7 & Specialized & $11 / 2$ & $11 / 3$ & + & + & $?$ \\
$G_{16}$ & 600 & 5 & Specialized & $11 / 0$ & $11 / 0$ & $?$ & + & $?$ \\
$G_{20}$ & 360 & 3 & Specialized & $2 / 0$ & $2 / 0$ & + & + & $?$ \\
$G_{21}$ & 720 & 5 & Specialized & $6 / 0$ & $6 / 0$ & $?$ & + & $?$ \\
$G_{22}$ & 240 & 2 & + & $1 / 0]$ & $4 / 0$ & + & + & $?$ \\
\hline
\end{tabular}

TABLE 9. Hecke algebras for three-dimensional primitive groups.

\begin{tabular}{cccccccc}
\hline$W$ & $|W|$ & $|\mathbf{u}|$ & Algebra & $t\left(T_{\mathbf{w}}\right) \neq 0$ & $t\left(T_{\mathbf{w}}{ }^{-1} \boldsymbol{\pi}\right) \neq 0$ & Good & Rank [18] \\
\hline$G_{24}, P_{1}$ & 336 & 2 & + & 0 & 0 & + & + \\
$G_{24}, P_{2}$ & & 2 & + & $3 / 0$ & $4 / 0$ & + & + \\
$G_{24}, P_{3}$ & & 2 & + & 0 & 0 & + & + \\
$G_{25}$ & 648 & 3 & Specialized & 0 & 0 & + & + \\
$G_{26}$ & 1296 & 5 & Specialized & 0 & 0 & $?$ & + \\
$G_{27}, P_{1}$ & 2160 & 2 & + & $1 / 1$ & $30 / 6$ & $?$ & \\
$G_{27}, P_{2}$ & & 2 & + & $41 / 1$ & $97 / 28$ & $?$ & + \\
$G_{27}, P_{3}$ & & 2 & + & $31 / 9$ & $44 / 24$ & $?$ & + \\
$G_{27}, P_{4}$ & & 2 & + & $19 / 2$ & $42 / 1$ & $?$ & \\
\hline
\end{tabular}




\subsection{Checking that the section is good}

To check (2.8) we avoid having to give an expression for $\mathbf{w}^{-1} \boldsymbol{\pi}$ in terms of the generators by using that $\chi\left(T_{\mathbf{w}^{-1} \boldsymbol{\pi}}\right)=\chi\left(\left(T_{\mathbf{w}}\right)^{-1}\right) \omega_{\chi}\left(T_{\boldsymbol{\pi}}\right)$, where $\omega_{\chi}\left(T_{\boldsymbol{\pi}}\right)$ is easy to compute using, for example, the formula $[8,1.22]$.

In the column 'Good' in Tables 8 and 9 we have recorded with a ' + ' if we could check that the section built in the previous subsection is good, and satisfies the assumptions of Proposition 2.10, thus providing an $A$-basis of $\mathcal{H}(W, \mathbf{u})$.

\subsection{Tables}

In Tables 8 and 9 we collect the computational results that we have obtained so far, together with results from an unpublished note of Müller [18]. Here, we write 'Specialized' in the column 'Algebra' when we had to do the computation with parameters specialized to prime powers.

Müller used Linton's vector enumerator to construct the regular representation of some cyclotomic Hecke algebras $\mathcal{H}(W)$. From that, he is able to verify Conjecture 2.2(a) in several cases by exhibiting an $A$-basis, marked by a ' + ' in the column 'Rank' of our tables. Furthermore, he can construct a symmetrizing form over $A$ satisfying Conjecture 2.2(b) in the cases marked ' + ' in the column 'Form'.

Neither Müller nor we have been able to check any of the cases $G_{17}, G_{18}, G_{19}$, for which the number of parameters is at least seven and the order of $W$ at least 1200 .

In his computations, Müller only looked at the presentations $P_{2}, P_{3}$ for the group $G_{27}$.

Acknowledgements. The authors thank the Mathematical Sciences Research Institute, Berkeley, and the Isaac Newton Institute for Mathematical Sciences, Cambridge, for their hospitality during the preparation of parts of this work.

\section{References}

1. S. ARIKI, 'Representation theory of a Hecke algebra of $G(r, p, n)$ ', J. Algebra 177 (1995) 164-185.

2. S. ARIKI and K. KOIKE, 'A Hecke algebra of $(Z / r Z)>\mathfrak{S}_{n}$ and construction of its irreducible representations', Adv. Math. 106 (1994) 216-243.

3. D. Bessis, 'Zariski theorems and diagrams for braid groups', Invent. Math 145 (2001) 487-507.

4. D. Bessis, 'Finite complex reflection arrangements are $K(\pi, 1)$ ', 2008, arXivmath/0610777.

5. D. Bessis and J. Michel, 'Explicit presentations for exceptional braid groups', Experiment. Math. 13 (2004) 257-266.

6. K. Bremke and G. Malle, 'Reduced words and a length function for $G(e, 1, n)$ ', Indag. Math. 8 (1997) 453-469.

7. M. Broué and G. Malle, 'Zyklotomische Heckealgebren', Astérisque 212 (1993) 119-189.

8. M. Broué, G. Malle and J. Michel, 'Towards spetses I', Transform. Groups 4 (1999) 157-218.

9. M. Broué, G. Malle and R. Rouquier, 'Complex reflection groups, braid groups, Hecke algebras', J. reine angew. Math. 500 (1998) 127-190.

10. R. Dipper, G. James and A. Mathas, 'Cyclotomic q-Schur algebras', Math. Z. 229 (1998) 385-416.

11. P. Etingof and E. RAins, 'Central extensions of preprojective algebras, the quantum Heisenberg algebra, and 2-dimensional complex reflection groups', J. Algebra 299 (2006) 570-588.

12. A. Gyoja, 'On the existence of a $W$-graph for an irreducible representation of a Coxeter group', J. Algebra 86 (1984) 422-438.

13. G. MAlle, 'Degrés relatifs des algèbres cyclotomiques associées aux groupes de réflexions complexes de dimension deux', Finite reductive groups: related structures and representations, Progress in Mathematics 141 (Birkhäuser, Basel, 1997) 311-332.

14. G. MAlle, 'On the rationality and fake degrees of characters of cyclotomic algebras', J. Math. Sci. Univ. Tokyo 6 (1999) 647-677.

15. G. Malle, 'On the generic degrees of cyclotomic algebras', Represent. Theory 4 (2000) 342-369.

16. G. Malle and A. Mathas, 'Symmetric cyclotomic Hecke algebras', J. Algebra 205 (1998) 275-293.

17. J. MicheL, 'The GAP-part of the Chevie system', GAP 3-package available for download from http://people.math.jussieu.fr/ jmichel/chevie/chevie.html.

18. J. MüLLER, 'On exceptional cyclotomic algebras', Private communication, June 2004. 
Gunter Malle

FB Mathematik

TU Kaiserslautern

Postfach 3049,

67653 Kaiserslautern

Germany

malle@mathematik.uni-kl.de
Jean Michel

CNRS

Université Paris VII

175, rue du Chevaleret, 75013 Paris

France

jmichel@math.jussieu.fr 